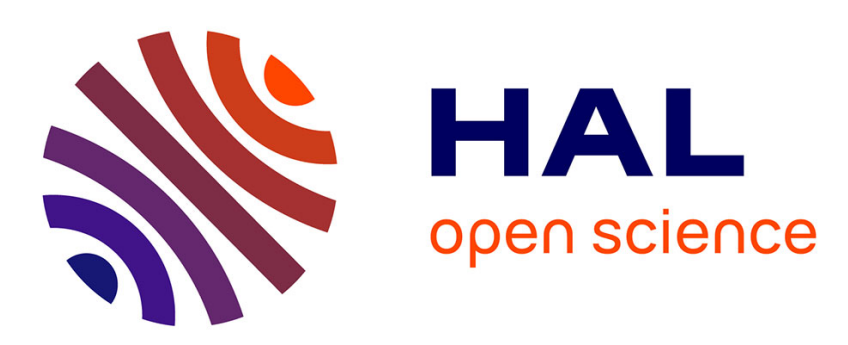

\title{
Gust Load Alleviation: Identification, Control, and Wind Tunnel Testing of a 2-D Aeroelastic Airfoil
}

Charles Poussot-Vassal, Fabrice Demourant, Arnaud Lepage, Dominique Le Bihan

\section{- To cite this version:}

Charles Poussot-Vassal, Fabrice Demourant, Arnaud Lepage, Dominique Le Bihan. Gust Load Alleviation: Identification, Control, and Wind Tunnel Testing of a 2-D Aeroelastic Airfoil. IEEE Transactions on Control Systems Technology, 2016, p. 1-14. 10.1109/TCST.2016.2630505 . hal-01461380

\section{HAL Id: hal-01461380 \\ https://hal.science/hal-01461380}

Submitted on 8 Feb 2017

HAL is a multi-disciplinary open access archive for the deposit and dissemination of scientific research documents, whether they are published or not. The documents may come from teaching and research institutions in France or abroad, or from public or private research centers.
L'archive ouverte pluridisciplinaire HAL, est destinée au dépôt et à la diffusion de documents scientifiques de niveau recherche, publiés ou non, émanant des établissements d'enseignement et de recherche français ou étrangers, des laboratoires publics ou privés. 


\title{
Gust load alleviation: identification, control and wind tunnel testing of a $2 \mathrm{D}$ aeroelastic airfoil
}

\author{
C. Poussot-Vassal, F. Demourant, A. Lepage and Dominique Le Bihan
}

\begin{abstract}
One important element in the progression of aircraft environmental impact reduction is to reduce their overall weight (without impacting other consumption-oriented performance index, such as drag). In addition to the numerous work conducted in material and structural engineering, from a control viewpoint, this challenge is strongly connected to the need of the development and assessment of dedicated load control strategies in response to gust disturbances. Indeed, the load factors due to gust are considered as a sizing criteria during the aircraft conception steps and require specific verification according to the certification process. To this end, a dedicated experimental research program based on Wind Tunnel (WT) campaigns has been carried out. More specifically, the paper contributions are twofold: $(i)$ to identify the gust load effect using two different versatile frequency-domain techniques, namely the Loewner interpolation and a modified subspace approach, and (ii) to design and implement an active closed-loop control to alleviate the gust main effect. The entire procedure is validated in a wind tunnel set-up, involving a gust generator device and a 2D aeroelastic airfoil, for varying configuration travelling from sub to transonic airflow and varying angles of attack, emphasizing the effectiveness and robustness of the overall approach.
\end{abstract}

Index Terms-Gust load control, Rational interpolation, Subspace, Robust control, Wind tunnel test.

\section{INTRODUCTION}

\section{A. Context and experimental set-up}

$\mathbf{T}$ HE topic of this paper concerns the design and the experimental validation of an active closed-loop gust load alleviation controller applied on a $2 D$ wing in wind tunnel testing. Real experiments were achieved and validated within the ONERA Wind Tunnel (WT) facility. Real experiments were achieved and validated within the ONERA S3Ch Wind Tunnel (WT) facility for various test conditions - Angles of Attack (AoA) and Mach numbers ${ }^{1}$. The contributions made available in this paper encapsulate many research fields, going from mechanical design to unsteady aerodynamic characterisation of gust, including signal processing and aeroelasticity studies. However, the present paper is mainly focussed on the control engineering side, and more specifically, the open-loop dynamical model identification and closed-loop control design steps. An objective is to provided a rationalization of the gust control design.

From this control viewpoint, it is worth mentioning that the gust load alleviation problem has driven a considerable attention in the literature to substantially enhance load aircraft

ONERA - The French Aerospace Lab, F-31055 Toulouse, France.

Corresponding author: C. Poussot-Vassal, charles.poussot-vassal@onera.fr

${ }^{1}$ http://www.onera.fr/en/news/gust-effect-control-a-european-first-at-onera (project webpage). performances, see e.g. [1], [2], [3], [4], [5], [5], [6], [7], [8], [9], [10] $]^{2}$. However a few involving real wind tunnel tests, and, to the authors' best of knowledge, most of them refer to tests performed in the low speed regime [11], [12]. The originality of this work relies on the WT investigations achieved up to the transonic regime covering from the generation to the active alleviation of gust loads, which makes the results of this paper particularly relevant and the experimental demonstrations quasi-unique $[13]^{3}$. In this work, the wind tunnel investigations cover the transonic air speed, which is the operation cruise regime of most modern aircraft and the most complex aerodynamic range. In aerodynamics, transonic flow involves sub and supersonic local flow in the same flow field (airflows surrounding an aircraft are locally below, at, and above the speed of sound). The transonic condition refers to compressibility effects and is characterised by discontinuities and nonlinear effects (drag divergence, shock wave, flow separation...). This constitutes the main challenge addressed in this work.

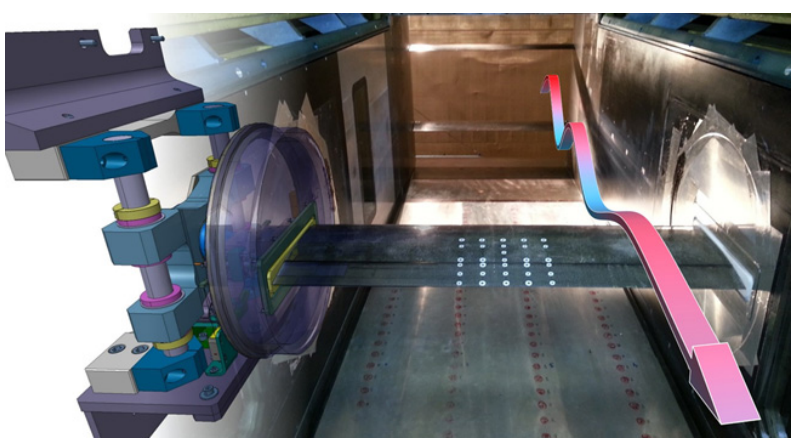

Fig. 1. Experimental set-up (internal view of the WT). Controlled 2D airfoil and electromechanical mechanism structure (foreground), gust generator (background) and air flow stream (graphical arrow).

As illustrated on Figure 1, the experimental research mean involved in this work consists of two main parts implemented within the WT facility. The first one is dedicated to the generation of the gust field, composed of two airfoils, and is called the gust generator. The second one is the $2 D$ wing model which aerodynamic and aeroelastic behaviours were studied and tailored with objective of assessing the

\footnotetext{
${ }^{2}$ Indeed, since it is a sizing criteria during the conception phase for every aircraft, gust load alleviation plays a crucial role in aeronautics and has motivated many academic and industrial researches.

${ }^{3}$ Transonic case lead to turbulent and contaminated nonlinear flow, resulting in drag and a loss of efficiency. This phenomena is widely studied in flow dynamics (see e.g. [14])
} 
gust load alleviation functions. A schematic overview of the experimental set-up is also shown in Figure $2^{4}$.

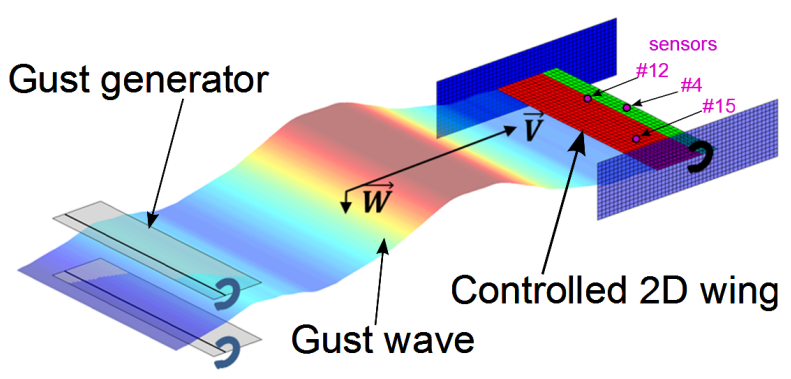

Fig. 2. Experimental set-up schematic view: gust wave (varying coloured surface) generated by the gust generator (left horizontal wings) attacking the controlled 2D airfoil (right horizontal wing). The airfoil is equipped with a movable surface (green) and 20 sensors (three of them, \#4, \#12, \#15, are materialized by pink dots, and will be used either as control measurements or performance objectives). $\vec{v}$ is the wind stream vector of the wind tunnel and $\vec{w}$ is the vertical gust vector caused by the gust generator.

The gust generator (or disturbance generator). From an air flow stream generated by the WT, the gust generator aims at superposing parametric vertical gust velocities. This is achieved by the adequate actuation of the two airfoils performed by four servo-hydraulic jacks which synchronously rotate the two airfoils (i.e. pitch dynamic motions about the quarter chord point). Each airfoil is driven at its two sides and the actuation device includes a security system that stops the actuation whenever the two roots of one airfoil are not at the same angular position, thus preventing any unwanted torsional deformation of the airfoils. The two airfoils are made of an optimized architecture that combines carbon reinforced skins and metallic parts to reduce inertia and allow high frequencies of actuation under heavy aerodynamic loads. In addition to inertial and strength constraints, the dynamic behaviour of the airfoils was tuned such that the frequency bandwidth of the gust generator is about $100 \mathrm{~Hz}$.

The controlled 2D airfoil (controlled system). It represents the considered system to be first identified and then controlled. The model consists in a 2D airfoil mounted between the walls of the WT sections with specific boundary conditions allowing two flexible degrees of freedom: pitch and plunge/heave motions. Is is equipped with a movable full span trailing edge control surface (green area on Figure 2) which is actuated on each side by a high torque - high speed hydraulic actuator. This wing is symmetrically actuated to avoid torsional effects. The actuator's bandwidth is of $100 \mathrm{~Hz}$, but in the closed-loop set-up, will be limited to $50 \mathrm{~Hz}$ to focus on the first structural mode (for this first attempt of active control demonstration). The wing is also equipped with 20 acceleration sensors located on the wingspan (some stations are materialized by pink marks on Figure 2). Figure 3 illustrates typical open-loop frequency responses (transfer gain) of the vertical acceleration $\ddot{z}_{\# 15}$ at station \#15 obtained in WT facility at four different configurations/combinations of Mach number and

\footnotetext{
${ }^{4}$ The experimental set-up was designed and manufactured through an ONERA - Aviation Design collaboration, Aviation Design being a French S.M.E. winner of a Call for Proposal. Additional results on the facility and aeroeslasticity results can be found in [15].
}

$\mathbf{A o A}$, in response to both gust disturbance $w(t)$ (left frame) and movable control surface input $u(t)$ (right frame). The correspondence between each frequency amplification and the associated physical motions of the wing (obtained during the dynamic characterisation of the wing in laboratory), are also shown.

Gust load alleviation control objective. Accordingly to the experimental device and to standard aeronautical requirements, the main problem addressed here is, for all the considered $n_{s}$ Mach numbers/AoA combinations, to reduce the gust effect $w(t)$ on the wing vertical acceleration at station \#15, i.e. $\ddot{z}_{\# 15}(t)$ (e.g. reduce Figure 3 left frame heave peaks) by an adequate actuation of the movable control surface input $u(t)^{5}$. More specifically, attention will be given in disturbance attenuation over the frequency range $\Omega=\left[\begin{array}{ll}15 & 50\end{array}\right] 2 \pi \mathrm{rad} / \mathrm{s}$. From the control designer point of view and in a linear framework, by denoting $\mathcal{S}^{(i)}\left(i=1, \ldots, n_{s}\right)$ as the nominal open-loop model of system for the $i$ th Mach number/AoA configuration, with associated transfer function $\mathbf{H}^{(i)}(s)$, this objective can be recast as finding $\mathbf{K}^{\star}(s)$ such that,

$$
\mathbf{K}^{\star}(s):=\underset{\mathbf{K}(s) \in \mathcal{H}_{2}}{\arg \min }\left\|\mathcal{F}_{l}\left(\mathbf{H}^{(i)}(s), \mathbf{K}(s)\right)_{w \rightarrow \ddot{z}}\right\|_{\mathcal{H}_{\infty}},
$$

for all $i=1, \ldots, n_{s}$, where $\mathbf{K}^{\star}(s)$ is the linear time invariant optimal controller that minimizes the $\mathcal{H}_{\infty}$-norm ${ }^{6}$ of the lower fractional interconnection $\left(\mathcal{F}_{l}\left(\mathbf{H}^{(i)}(s), \mathbf{K}^{\star}(s)\right)\right)$ between the system $\mathbf{H}(s)$ and aforementioned active controller. Problem (1) means that one is looking for a strictly proper and stable controller $\mathbf{K}^{\star}(s)$ that (stabilizes and) attenuates the first peak, where gust generally occurs. Therefore, throughout the paper, focus on vertical accelerations reduction will be addressed. In the control literature, this objective can also be viewed as an active damping problem.

Remark 1 (WT experiments): It is worth mentioning that, before obtaining the open-loop data, more than hundreds of experiments have been performed during two months to calibrate and adjust the gust generator, wing profile and perform adequate measurements. Then, as rooted on the numericalbased open-loop models and robust control controller synthesis (described in this paper), the WT closed-loop experiments have been performed in two days. This consideration is very important since WT experiments are relatively expensive.

\section{B. Contributions}

The main contributions of this work are twofold: first $(i)$ in successfully identifying the dynamical model through two advanced frequency-domain methods: one grounded on the Loewner interpolation framework (see [17], [18], [19]) and the other, based on an innovative subspace formulation including eigenvalues constraints based on LMI regions ${ }^{7}$. Second, (ii)

\footnotetext{
${ }^{5}$ Indeed, in the context of this study, this attenuation will lead to wing root bending moment minimization which is immediately linked to the mass at the wing/fuselage junction. In a more general framework of the actual civilian aircraft research, one objective is to the increase of the laminar flow working zone which are central objectives in consumption optimization [16].

${ }^{6}$ The $\mathcal{H}_{\infty}$-norm, with appropriated weighting functions can also minimizes the root mean square over the frequency range $\Omega$ (see Section III).

${ }^{7}$ It is shown that the two approaches are complementary since one is involved for the synthesis, while the other for numerical validation phases.
} 


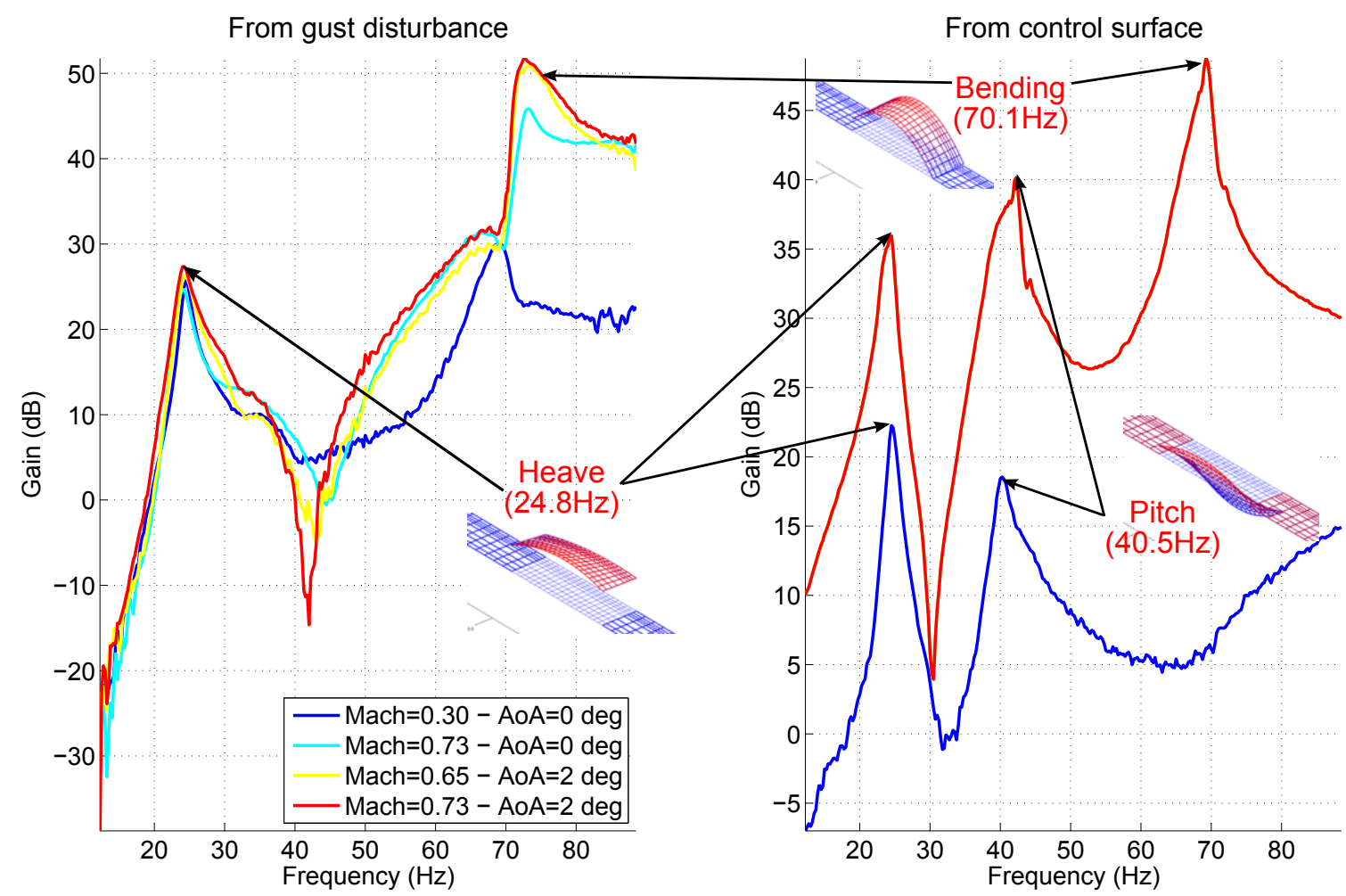

Fig. 3. Open-loop frequency responses of the vertical acceleration $\ddot{z}_{\# 15}$ of the wing at station \#15 obtained in WT experiments in response to the gust disturbance (left) and to the movable surface actuator (right). Responses for four Mach number/AoA configurations. Since it represents the transfer from the control actuator, right frame exhibit two transfer, only, one for each Mach number.

in synthesizing and implementing a gust load alleviation controller using a structured $\mathcal{H}_{\infty}$ optimization criteria approach (see [20]). As a glimpse of the obtained results, Figure 4 illustrates the vertical acceleration attenuation obtained in WT, for constant AoA and for various Mach numbers, in response to varying frequency gust wave disturbances. Figure $4 \mathrm{em}$ phasizes the benefit of the proposed identification and control procedure, leading to the first frequency peak attenuation, while keeping the rest of the frequency response unchanged (see details in the rest of the paper).

Remark 2 (Media material, at Mach number 0.73 and AoA 0 degree): in addition to the present paper, a video is also made available at the following address: http://www.onera.fr/en/news/gust-effect-controla-european-first-at-onera, illustrating the attenuation of the displacement amplifications in response to a gust disturbance at the characteristic frequency $25 \mathrm{~Hz}$ (heave frequency), with and without control law, in a transonic configuration.

\section{Paper structure}

The rest of the paper is dedicated to the description and illustration of the applied methodology to achieve results of Figure 4. The paper is organized in a top down framework. First, in Section II, two frequency-domain methods are presented and applied to the considered system to identify the linear dynamical models that accurately captures the dynamics in response to gust disturbance and surface deflection. Then, Section III is devoted to the design of the dedicated robust active gust load controller, followed by some simulations and

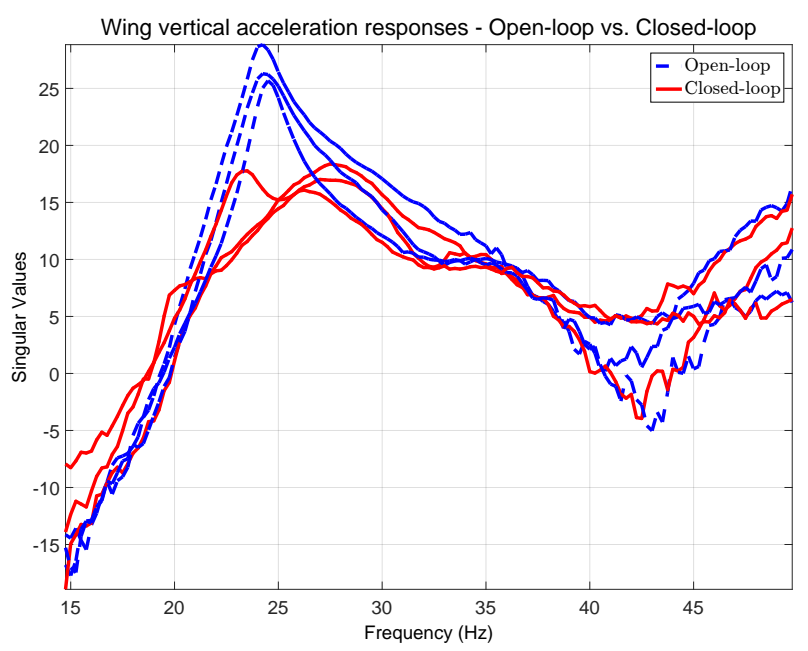

Fig. 4. Wind tunnel experimental data results. Comparison of the frequency response of the singular value of the vertical accelerations at station \#15, for varying Mach number, with AoA of 0 degree. Open loop: dashed blue. Closed-loop: solid red.

experimental results (illustrating both the consistency between numerical and experimental data, and effectiveness of the methodology). Conclusions are finally given in Section IV.

\section{Mathematical notations}

Let us consider a stable Multiple Input/Multiple Output (MIMO) linear dynamical system $\mathcal{S}$, with $n_{u}\left(\right.$ resp. $\left.n_{y}\right) \in \mathbb{N}^{*}$ 
input(s) (resp. output(s)), represented by its $n$-th order transfer function $\mathbf{H} \in \mathbb{C}^{n_{y} \times n_{u}}$, where $\mathbf{H}(s)=\mathbf{C}(s \mathbf{E}-\mathbf{A})^{-1} \mathbf{B}+\mathbf{D}$. Its associated realisation reads $\mathcal{S}:(\mathbf{E}, \mathbf{A}, \mathbf{B}, \mathbf{C}, \mathbf{D})$,

$$
\mathcal{S}: \mathbf{E} \dot{\mathbf{x}}(t)=\mathbf{A} \mathbf{x}(t)+\mathbf{B} \mathbf{u}(t), \mathbf{y}(t)=\mathbf{C} \mathbf{x}(t)+\mathbf{D} \mathbf{u}(t) .
$$

The input vector used here gathers the gust disturbance and control deflection as $\mathbf{u}(t)=[w(t) u(t)]$. The considered output vector gathers three acceleration sensors at stations \#4, \#12 and $\# 15$, i.e. $\mathbf{y}(t)=\left[\ddot{z}_{\# 4}(t) \ddot{z}_{\# 12}(t) \ddot{z}_{\# 15}(t)\right]$. Then, the approximate model, or reduced order model of order $r \ll n$, will be denoted as $\hat{\mathbf{H}}(s)=\hat{\mathbf{C}}(s \hat{\mathbf{E}}-\hat{\mathbf{A}})^{-1} \hat{\mathbf{B}}+\hat{\mathbf{D}}$. Its associated realisation reads $\hat{\mathcal{S}}:(\hat{\mathbf{E}}, \hat{\mathbf{A}}, \hat{\mathbf{B}}, \hat{\mathbf{C}}, \hat{\mathbf{D}})$,

$$
\hat{\mathcal{S}}: \hat{\mathbf{E}} \dot{\hat{\mathbf{x}}}(t)=\hat{\mathbf{A}} \hat{\mathbf{x}}(t)+\hat{\mathbf{B}} \mathbf{u}(t), \hat{\mathbf{y}}(t)=\hat{\mathbf{C}} \hat{\mathbf{x}}(t)+\hat{\mathbf{D}} \mathbf{u}(t) .
$$

\section{OPEN-LOOP MODEL IDENTIFICATION AND VALIDATION PHASE}

As illustrated on Figures 3 and 4, the ONERA experimental WT facility allows the generation of accurate gust disturbances at varying frequencies (and amplitudes). Consequently, it is possible to obtain the frequency-domain responses $\boldsymbol{\Phi}_{i} \in$ $\mathbb{C}^{n_{y} \times n_{u}}$ for different frequency samples $\omega_{i}(i=1, \ldots, N)$. Then, one can write:

$$
\mathbf{H}\left(\imath \omega_{i}\right)=\boldsymbol{\Phi}_{i},
$$

where $\mathbf{H}$ is the $\operatorname{exact}^{8}$ transfer function of the system. Based on the couple $\left(\omega_{i}, \boldsymbol{\Phi}_{i}\right)_{i=1}^{N}$, the main purpose of this section is to obtain a low-order rational LTI model of the form, $\hat{\mathbf{H}}(s)=\hat{\mathbf{C}}(s \hat{\mathbf{E}}-\hat{\mathbf{A}})^{-1} \hat{\mathbf{B}}+\hat{\mathbf{D}} \in \mathcal{H}_{\infty}^{n_{y} \times n_{u}}$, with realisation $\hat{\mathcal{S}}:(\hat{\mathbf{E}}, \hat{\mathbf{A}}, \hat{\mathbf{B}}, \hat{\mathbf{C}}, \hat{\mathbf{D}})$, defined as:

$$
\hat{\mathcal{S}}: \hat{\mathbf{E}} \dot{\hat{\mathbf{x}}}(t)=\hat{\mathbf{A}} \hat{\mathbf{x}}(t)+\hat{\mathbf{B}} \mathbf{u}(t), \hat{\mathbf{y}}(t)=\hat{\mathbf{C}} \hat{\mathbf{x}}(t)+\hat{\mathbf{D}} \mathbf{u}(t),
$$

that well matches the frequency samples $\left(\omega_{i}, \boldsymbol{\Phi}_{i}\right)_{i=1}^{N}$ and hopefully reproduces the real transfer $\mathbf{H}$ (in other words, $\hat{\mathbf{y}}$ should accurately reproduce $\mathbf{y}$ ). Let $\hat{\mathbf{x}}(t) \in \mathbb{R}^{r}, \mathbf{u}(t) \in \mathbb{R}^{n_{u}}$ and $\hat{\mathbf{y}}(t) \in \mathbb{R}^{n_{y}}$ be the states, inputs and outputs vectors, respectively. To this aim, two approaches have been experimented: the first one is based on the Loewner framework (Section II-A), and the second one, more innovative, on the subspace one with LMI constraints (Section II-B). Both approaches are used in this work for different purpose. While the second approach will be used for the control design step, the former one is involved for numerical validation purpose ${ }^{9}$.

\section{A. First approach: frequency-domain matrix function interpo- lation and model approximation}

The first approach is based on the interpolatory framework well defined in [17], [19], involving the Loewner matrices, and recalled hereafter. It is composed of two steps: first an exact rational model interpolation, followed by a reduction procedure $^{10}$. To this aim, let us first partition the collected

\footnotetext{
${ }^{8}$ By exact, one intends the unknown, real exact function that describes the frequency responses obtained in WT facility. Of course, any model reproducing the data collected in experiment will be called exact if perfect match is ensured. However, every model can still be amended or questioned, and the search for the perfect model is hopelessly unachievable (and is not necessary for control design).

${ }^{9}$ For a more detailed qualitative comparison between the Loewner and subspace approach, reader is invited to refer to [21].

${ }^{10}$ Most of the results here described are available in [18]. A very complete survey is also available in [19].
}

data $\left(\omega_{i}, \boldsymbol{\Phi}_{i}\right)_{i=1}^{N}$ in two disjoint sets as follows $(N=q+k)$ :

$$
\begin{aligned}
\imath\left[\omega_{1}, \ldots, \omega_{N}\right] & =\left[\mu_{1}, \ldots, \mu_{q}\right] \cup\left[\lambda_{1}, \ldots, \lambda_{k}\right] \\
{\left[\boldsymbol{\Phi}_{1}, \ldots, \boldsymbol{\Phi}_{N}\right] } & =\left[\tilde{\mathbf{v}}_{1}, \ldots, \tilde{\mathbf{v}}_{q}\right] \cup\left[\tilde{\mathbf{w}}_{1}, \ldots, \tilde{\mathbf{w}}_{k}\right] .
\end{aligned}
$$

Then, define $\mathbf{l}_{j} \in \mathbb{C}^{n_{y} \times 1}(j=1, \ldots, q)$ and $\mathbf{r}_{i} \in \mathbb{C}^{n_{u} \times 1}$ $(i=1, \ldots, k)$ the $q$ left and $k$ right tangential directions. Using these tangential directions, let us define $\mathbf{v}_{j}^{*}=\mathbf{l}_{j}^{*} \tilde{\mathbf{v}}_{j} \in \mathbb{C}^{1 \times n_{u}}$ and $\mathbf{w}_{i}=\tilde{\mathbf{w}}_{i} \mathbf{r}_{i} \in \mathbb{C}^{n_{y} \times 1}$ the left and right tangential data directions, respectively. Then, considering the right or column data and the left or row data, and assuming that $\lambda_{i}$ and $\mu_{j}$, are distinct, the right interpolation data are organized as:

$$
\left.\begin{array}{rl}
\boldsymbol{\Lambda} & =\operatorname{diag}\left(\lambda_{1}, \ldots, \lambda_{k}\right) \in \mathbb{C}^{k \times k} \\
\mathbf{R} & =\left[\mathbf{r}_{1} \ldots \mathbf{r}_{k}\right] \in \mathbb{C}^{n_{u} \times k} \\
\mathbf{W} & =\left[\begin{array}{lll}
\mathbf{w}_{1} & \ldots & \mathbf{w}_{k}
\end{array}\right] \in \mathbb{C}^{n_{y} \times k}
\end{array}\right\},
$$

and the left interpolation data are given as,

$$
\left.\begin{array}{rl}
\mathbf{M} & =\operatorname{diag}\left(\mu_{1}, \ldots, \mu_{q}\right) \in \mathbb{C}^{q \times q} \\
\mathbf{L}^{*} & =\left[\mathbf{l}_{1} \ldots \mathbf{l}_{q}\right] \in \mathbb{C}^{n_{y} \times q} \\
\mathbf{V}^{*} & =\left[\mathbf{v}_{1} \ldots \mathbf{v}_{q}\right] \in \mathbb{C}^{n_{u} \times q}
\end{array}\right\}
$$

Based on (7) and (8), the corresponding Loewner $\mathbb{L} \in \mathbb{C}^{q \times k}$ and shifted Loewner $\mathbb{L}_{\sigma} \in \mathbb{C}^{q \times k}$ matrices, are constructed as follows, for $i=1, \ldots, k$ and $j=1, \ldots, q$ :

$$
\begin{aligned}
& {[\mathbb{L}]_{j, i}=\frac{\mathbf{v}_{j}^{*} \mathbf{r}_{i}-\mathbf{l}_{j}^{*} \mathbf{w}_{i}}{\mu_{j}-\lambda_{i}}=\frac{\mathbf{l}_{j}^{*}\left(\mathbf{H}\left(\mu_{j}\right)-\mathbf{H}\left(\lambda_{i}\right)\right) \mathbf{r}_{i}}{\mu_{j}-\lambda_{i}}} \\
& {\left[\mathbb{L}_{\sigma}\right]_{j, i}=\frac{\mu_{j} \mathbf{v}_{j}^{*} \mathbf{r}_{i}-\lambda_{i} \mathbf{l}_{j}^{*} \mathbf{w}_{i}}{\mu_{j}-\lambda_{i}}=\frac{\mathbf{l}_{j}^{*}\left(\mu_{j} \mathbf{H}\left(\mu_{j}\right)-\lambda_{i} \mathbf{H}\left(\lambda_{i}\right)\right) \mathbf{r}_{i}}{\mu_{j}-\lambda_{i}},}
\end{aligned}
$$

which satisfy the following Sylvester equations:

$$
\begin{aligned}
\mathbf{L} \boldsymbol{\Lambda}-\mathbf{M L} & =\mathbf{L W}-\mathbf{V R}, \\
\mathbb{L}_{\sigma} \boldsymbol{\Lambda}-\mathbf{M L}_{\sigma} & =\mathbf{L W} \boldsymbol{\Lambda}-\mathbf{M V R} .
\end{aligned}
$$

Exact rational interpolation. Based on the above data and tangential directions, the interpolation problem can be stated as follows:

Problem 1 (Data-driven interpolation [17]): Given left interpolation driving frequencies $\left\{\mu_{i}\right\}_{i=1}^{q} \in \mathbb{C}$ with left output or tangential directions $\left\{\mathbf{l}_{i}\right\}_{i=1}^{q} \in \mathbb{C}^{n_{y}}$, producing the left responses $\left\{\mathbf{v}_{i}\right\}_{i=1}^{q}$ and right interpolation driving frequencies $\left\{\lambda_{i}\right\}_{i=1}^{k} \in \mathbb{C}$ with right input or tangential directions $\left\{\mathbf{r}_{i}\right\}_{i=1}^{k} \in \mathbb{C}^{n_{u}}$, producing the right responses $\left\{\mathbf{w}_{i}\right\}_{i=1}^{k}$, find a (low order) system $\hat{\mathcal{S}}$ that such that the resulting transfer function $\hat{\mathbf{H}}(s)$ is an (approximate) tangential interpolant of the data, i.e. satisfies the following left and right interpolation conditions:

$$
\left.\begin{array}{c}
\mathbf{l}_{j}^{*} \hat{\mathbf{H}}\left(\mu_{j}\right)=\mathbf{v}_{j}^{*} \\
\text { for } j=1, \ldots, q
\end{array} \text { and } \begin{array}{c}
\hat{\mathbf{H}}\left(\lambda_{i}\right) \mathbf{r}_{i}=\mathbf{w}_{i} \\
\text { for } i=1, \ldots, k
\end{array}\right\} .
$$

Problem 1 can be solved thanks to the following theorem, proposed by [17].

Theorem 1 (Loewner framework [17]): Given right and left interpolation data as in (7)-(8), and assume that $k=q$ and let $\left(\mathbb{L}_{\sigma}, \mathbb{L}\right)$ be a regular pencil where $\lambda_{i}$ or $\mu_{j}$ are (distinct and) not eigenvalue. The rational transfer function $\mathbf{H}(s)$, with realisation $\mathcal{S}:(\mathbf{E}, \mathbf{A}, \mathbf{B}, \mathbf{C}, 0)$ constructed as

$$
\mathbf{E}=-\mathbb{L}, \mathbf{A}=-\mathbb{L}_{\sigma}, \mathbf{B}=\mathbf{V} \text { and } \mathbf{C}=\mathbf{W},
$$


with transfer minimal descriptor function

$$
\mathbf{H}(s)=\mathbf{W}\left(\mathbb{L}_{\sigma}-s \mathbb{L}\right)^{-1} \mathbf{V},
$$

interpolates the left and right constraints (11).

Theorem 1 allows to obtain a model $\mathcal{S}:(\mathbf{E}, \mathbf{A}, \mathbf{B}, \mathbf{C}, 0)$, whose transfer function interpolates the left and right constraints as stated in Problem 1. Moreover, an important property of the Loewner matrix is that,

$$
\operatorname{rank}(\mathbb{L})=n \leq N,
$$

encodes the McMillian degree $n$ of the rational function interpolation. Therefore, the exact Loewner interpolation provides a realisation of a rational transfer function that exactly interpolates all the data $\left(\omega_{i}, \boldsymbol{\Phi}_{i}\right)_{i=1}^{N}$, with the minimal order realisation thanks to the Loewner matrix rank.

Approximate rational interpolation. Once an exact model $\mathcal{S}:(\mathbf{E}, \mathbf{A}, \mathbf{B}, \mathbf{C}, 0)$ with transfer function $\mathbf{H}(s)$ with McMillian degree of $n \leq N$, and which tangentially interpolates the data is available, to obtain a reduced order model $\hat{\mathcal{S}}$ of order $r \leq n$ that well approximates $\mathcal{S}$ one simply apply an SVD as follows:

$$
\mathbb{L}=\left[\begin{array}{ll}
\mathbf{Y}_{1} & \mathbf{Y}_{2}
\end{array}\right]\left[\begin{array}{ll}
\Sigma_{1} & \\
& \Sigma_{2}
\end{array}\right]\left[\begin{array}{l}
\mathbf{X}_{1}^{*} \\
\mathbf{X}_{2}^{*}
\end{array}\right],
$$

where $\Sigma_{1}=\operatorname{diag}\left(\sigma_{1}, \ldots, \sigma_{r}\right) \in \mathbb{R}^{r \times r}, \quad \Sigma_{2}=$ $\operatorname{diag}\left(\sigma_{r+1}, \ldots, \sigma_{n}\right) \in \mathbb{R}^{(n-r) \times(n-r)}$ (where $\sigma_{1} \geq \sigma_{2} \geq \cdots \geq$ $\left.\sigma_{n}\right)$ and $\mathbf{Y}_{1}, \mathbf{Y}_{2}, \mathbf{X}_{1}, \mathbf{X}_{2}$ are of appropriate dimensions. Then the reduced order model $\hat{\mathbf{H}}$ is simply obtained by the PetrovGalerkin projection $^{11}$ :

$\hat{\mathcal{S}}:(\hat{\mathbf{E}}, \hat{\mathbf{A}}, \hat{\mathbf{B}}, \hat{\mathbf{C}}, 0)=\left(-\mathbf{Y}_{1}^{*} \mathbb{L} \mathbf{X}_{1},-\mathbf{Y}_{1}^{*} \mathbb{L}_{\sigma} \mathbf{X}_{1}, \mathbf{Y}_{1}^{*} \mathbf{V}, \mathbf{W} \mathbf{X}_{1}, 0\right)$.

In the objective - here considered - of identifying a model that accurately reproduces the gust and actuator effects on the wing profile, the main interest in Theorem 1 is that it enables to both find the exact rational function $\mathbf{H}$ (and realisation $\mathcal{S})$ that matches all the frequency-domain data $\left(\omega_{i}, \boldsymbol{\Phi}_{i}\right)_{i=1}^{N}$ and, allows, at a very low additional cost, to obtain a good approximated model $\hat{\mathbf{H}}$ (and realisation $\hat{\mathcal{S}})^{12}$.

Remark 3 (About optimality conditions): The truncation performed when applying the SVD in (16) does not guarantee the so-called $\mathcal{H}_{2}$-optimality conditions of the projected model (see e.g. [22]). These conditions can be achieved through an adequate interpolation data $\left(\mu_{i}\right.$ and $\left.\lambda_{i}\right)$ selection, as explained in recent papers [25], [26], but not tested here. Nevertheless, as illustrated in the forthcoming applicative section, very accurate results are obtained.

Application to the WT data. For the considered application, Theorem 1 is then used with $N=292$ sampled data points $(k=q=146), n_{y}=3$ outputs (acceleration at stations \#4, \#12 and \#15) and $n_{u}=2$ inputs (wind gust and actuator) and $r=20$. First, exact interpolation is achieved with a

\footnotetext{
${ }^{11}$ Practical considerations about complex arithmetic are also available in [18], [19].

${ }^{12}$ Obviously, from the original model $\mathbf{H}$, other approximation methods might be applied to obtain $\hat{\mathbf{H}}$. For instance, the IRKA [22], DARPO [23], etc. However, this is another issue not covered in the present paper (see e.g. the MORE toolbox developed at ONERA [24] for numerical tools encoding these algorithms)
}

state-space model of order $n=292$. The vertical acceleration response corresponding to station \#15 is illustrated on Figure 5 , with red lines, providing a model that perfectly matches the measurements (blue points). Then, thanks to the Loewner matrix properties, a rank truncation is performed to reduce the model to an order $r=20$ (singular values decay of $\mathbb{L}$ is not provided for space limitations), illustrating the good matching (dash-dotted pink lines).

Figure 5 illustrates the very good matching of the proposed models for two distinct configurations (sub and transonic). However, at this stage, no property on the spectrum associated to the obtained exact and approximate models can be given (e.g. stability of the model cannot be guaranteed). Indeed, the models might result in unstable ones in both cases (exact and approximate), which can be an issue since, in practice, the system is stable. This limitation is tackled in the next sub-section though an approach that also uses the frequency domain data, but in a different framework.

\section{B. Second approach: frequency-domain subspace with $\mathbf{L M I}$ constraints}

Similarly to the Loewner framework, the subspace approaches are well appropriate to identify and approximate frequency-domain sampled data, by a state-space realisation as in (5) (here with $\mathbf{E}=I_{n}$ and $\mathbf{D}$ non necessarily null). However, as in the Loewner case, in its basic version, no constraint on the obtained model eigenvalues location can be enforced (e.g. stability constraint). The main result in this section is to propose a solution to constraint the poles of the identified model in a user-defined region using Linear Matrix Inequalities (LMI). As made clearer in what follows, these LMI regions can simply be stability domain or more complex regions (horizontal strip, ellipse etc.). To the authors' best of knowledge, in the subspace context, the stability constraint is treated in [27], [28], [29], [30], [31]. In [31] a specific formulation based on discrete Lyapunov function is involved to impose stability whereas in the approach presented here a more general formulation is proposed to take into account LMI regions poles location and not only the stability one. The approaches developed in [27], [28] can distort the solution and techniques proposed in [29], [30] are not direct and based on iterative algorithm. Here the proposed solution is direct without any tuning parameters for a large class of poles location, where the stability preservation is a particular configuration. That is why we will talk about LMI regions rather than stability domain. This point is illustrated in the application section by using an elliptic constraint to impose not only stability for discrete models but also to skip irrelevant and unrealistic under-damped poles.

Preliminary results on subspace methods. Fundamental subspace-based results are briefly recalled here. However additional information can be found in [27], [32] ${ }^{13}$. Let

\footnotetext{
${ }^{13}$ For clarity purpose, the noise-free case is considered, only. Reader is invited to refer to [27] for the noisy case.
} 

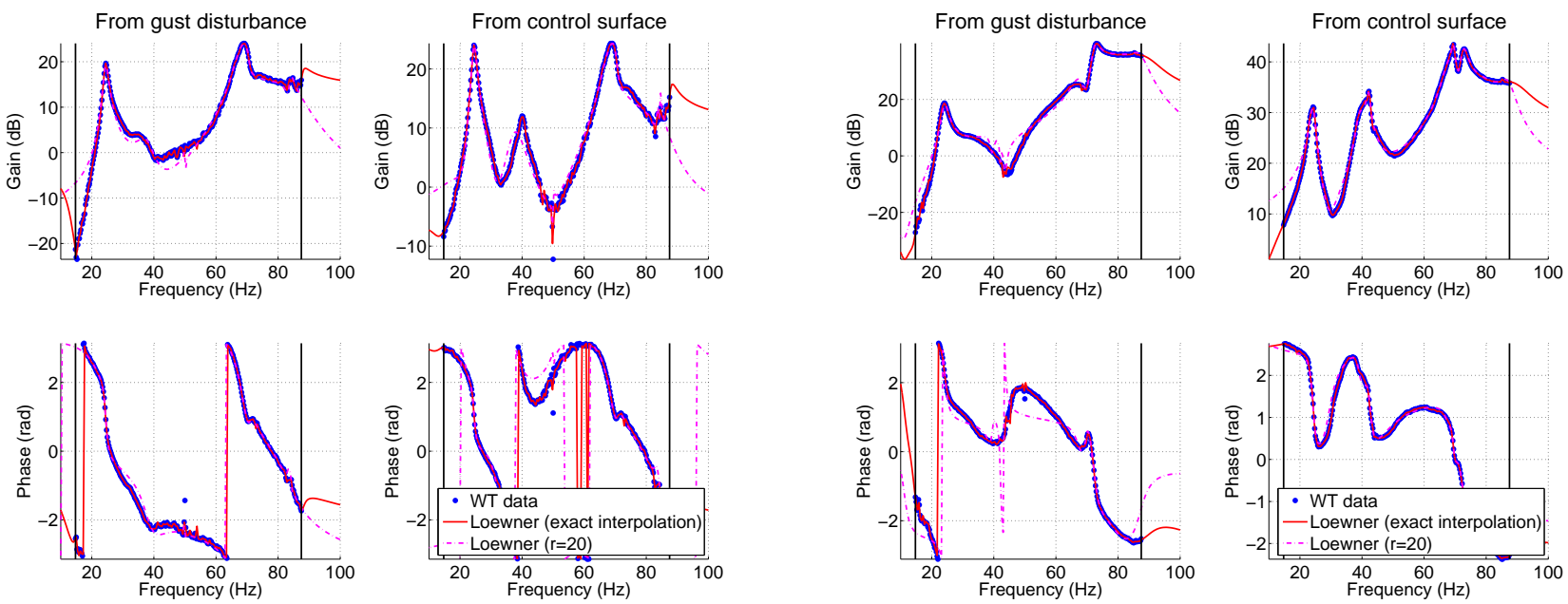

Fig. 5. First approach: comparison of the frequency response (gain and phase) of the vertical acceleration of the wing at station \#15 obtained in WT experiments (dotted blue) with models obtained via Loewner-based exact interpolation (solid red) and reduced with $r=20$ (dash-dotted magenta) interpolation. Left: Mach number 0.30 and AoA 0 degree. Right: Mach number 0.73 and AoA 0 degree. Vertical lines materialize the limit of the considered data set $\left(\omega_{i}, \boldsymbol{\Phi}_{i}\right)_{i=1}^{N}$.

us first consider the frequency domain discrete state-space representation:

$$
\begin{aligned}
e^{\imath \omega} \mathbf{X}(\omega) & =\mathbf{A X}(\omega)+\mathbf{B U}(\omega) \\
\mathbf{Y}(\omega) & =\mathbf{C X}(\omega)+\mathbf{D U}(\omega)
\end{aligned}
$$

where $\mathbf{X}(\omega) \in \mathbb{C}^{r}, \mathbf{U}(\omega) \in \mathbb{C}^{n_{u}}$ and $\mathbf{Y}(\omega) \in \mathbb{C}^{n_{y}}$ are the Fourier transform of the state $\mathbf{x}(t)$, the input $\mathbf{u}(t)$ and the output $\mathbf{y}(t)$ vectors, respectively. Let us then denote $\mathbf{X}_{m}\left(\omega_{i}\right)$ the state at frequency $\omega_{i}$ for an input vector $\mathbf{U}\left(\omega_{i}\right)$ with one in the $m$-th row and zero elsewhere $(i=1, \ldots, N$ and $m=$ $\left.1, \ldots, n_{u}\right)$, then (17) similarly reads as

$$
\begin{aligned}
e^{\imath \omega} \mathbf{X}(\omega) & =\mathbf{A X}(\omega)+\mathbf{B} \mathbf{U}(\omega) \\
\mathbf{G}(\omega) & =\mathbf{C X}^{c}(\omega)+\mathbf{D}
\end{aligned}
$$

where $\mathbf{X}^{c}(\omega)=\left[\mathbf{X}_{1}(\omega), \ldots, \mathbf{X}_{n_{u}}(\omega)\right] \in \mathbb{C}^{r \times n_{u}}$. Moreover, if a discrete frequency domain data set $\left(\omega_{i}, \boldsymbol{\Phi}_{i}\right)_{i=1}^{N}$ is considered then, one has $\mathbf{G}\left(\omega_{i}\right)=\boldsymbol{\Phi}_{i}$ and the following relation holds [27] (with $r+q \leq N$ and $q \geq r$ ):

$$
\mathbf{G}=\mathbf{O X}^{c}+\mathbf{\Gamma} \mathbf{W}
$$

where,

$$
\begin{aligned}
& \mathbf{G}=\left[\begin{array}{ccc}
\boldsymbol{\Phi}_{\mathbf{1}} & \ldots & \boldsymbol{\Phi}_{\mathbf{N}} \\
e^{\imath \omega_{1}} \boldsymbol{\Phi}_{\mathbf{1}} & \ldots & e^{\imath \omega_{N}} \boldsymbol{\Phi}_{\mathbf{N}} \\
\vdots & \ddots & \vdots \\
e^{\imath(q-1) \omega_{1}} \boldsymbol{\Phi}_{\mathbf{1}} & \ldots & e^{\imath(q-1) \omega_{N}} \boldsymbol{\Phi}_{\mathbf{N}}
\end{array}\right] \in \mathbb{C}^{n_{y} q \times n_{u} N}, \\
& \mathbf{X}^{c}=\left[\begin{array}{lll}
\mathbf{X}^{c}\left(\imath \omega_{1}\right) & \ldots & \mathbf{X}^{c}\left(\imath \omega_{N}\right)
\end{array}\right] \in \mathbb{C}^{r \times n_{u} N}
\end{aligned}
$$

and

$$
\mathbf{O}=\left[\begin{array}{c}
\mathbf{C} \\
\mathbf{C A} \\
\vdots \\
\mathbf{C A}^{q-1}
\end{array}\right] \in \mathbb{R}^{n_{y} q \times r}
$$

has full rank $r$ for all values $q \geq r$ and finally,

$$
\mathbf{W}=\left[\begin{array}{ccc}
I_{n_{u}} & \ldots & I_{n_{u}} \\
e^{\imath \omega_{1}} I_{n_{u}} & \ldots & e^{\imath \omega_{N}} I_{n_{u}} \\
\vdots & \ddots & \vdots \\
e^{\imath(q-1) \omega_{1}} I_{n_{u}} & \ldots & e^{\imath(q-1) \omega_{N}} I_{n_{u}}
\end{array}\right] \in \mathbb{C}^{n_{u} q \times n_{u} N}
$$

$$
\boldsymbol{\Gamma}=\left[\begin{array}{cccc}
\mathbf{D} & 0 & \ldots & 0 \\
\mathbf{C B} & \mathbf{D} & \ldots & 0 \\
\vdots & \vdots & \ddots & \vdots \\
\mathbf{C A}^{q-2} \mathbf{B} & \mathbf{C A}^{q-3} \mathbf{B} & \ldots & \mathbf{D}
\end{array}\right] \in \mathbb{R}^{n_{y} q \times n_{u} q}
$$

Relation (19) emphasizes that the extended observability matrix $\mathbf{O}$ (20), which depends on $\mathbf{A}$ and $\mathbf{C}$, can be written as a combination of inputs/outputs data $\mathbf{G}, \mathbf{X}^{c}$, and $\mathbf{W}$, only. Then, an elegant way to extract $\mathbf{A}$ and $\mathbf{C}$ matrices from $\mathbf{O}$, is to first apply the orthogonal projection $\mathbf{W}^{\perp}$ defined as

$$
\mathbf{W}^{\perp}=I-\mathbf{W}^{T}\left(\mathbf{W} \mathbf{W}^{T}\right)^{-1} \mathbf{W}
$$

to cancel the $\Gamma \mathbf{W}$ term ${ }^{14}$. Indeed, by right multiplying (19) with $\mathbf{W}^{\perp}$ as defined in (21), the following is obtained:

$$
\mathbf{G W}^{\perp}=\mathbf{O X}^{c} \mathbf{W}^{\perp} \text {. }
$$

Then, by denoting

$$
\left[\begin{array}{c}
\mathbf{W} \\
\mathbf{G}
\end{array}\right]=\left[\begin{array}{cc}
R_{11} & 0 \\
R_{21} & R_{22}
\end{array}\right]\left[\begin{array}{l}
Q_{1}^{T} \\
Q_{2}^{T}
\end{array}\right]=\mathbf{R Q},
$$

an effective way to extract $\mathbf{A}$ and $\mathbf{C}$ is to use a $\mathbf{Q R}$ and an SVD matrix decomposition of $\mathbf{G W}^{\perp} \in \mathbb{R}^{n_{y} q \times n_{u} q}$, and by noticing that:

$$
\begin{aligned}
\mathbf{G W}^{\perp}=R_{22} Q_{2}^{T} & =\hat{\mathbf{U}} \hat{\Sigma} \hat{\mathbf{V}}^{T} Q_{2}^{T} \\
& =\left[\begin{array}{ll}
\hat{\mathbf{U}}_{\mathbf{s}} & \hat{\mathbf{U}}_{\mathbf{o}}
\end{array}\right]\left[\begin{array}{cc}
\hat{\Sigma}_{s} & 0 \\
0 & \hat{\Sigma}_{o}
\end{array}\right]\left[\begin{array}{c}
\hat{\mathbf{V}}_{\mathbf{s}}^{T} \\
\hat{\mathbf{V}}_{\mathbf{o}}^{T}
\end{array}\right] Q_{2}^{T} .
\end{aligned}
$$

where $\hat{\Sigma}_{s}=\operatorname{diag}\left(\sigma_{1}, \ldots, \sigma_{r}\right) \in \mathbb{R}^{r \times r}$ and $\hat{\Sigma}_{o}=$ $\operatorname{diag}\left(\sigma_{r+1}, \ldots, \sigma_{n_{y} q}, 0\right) \in \mathbb{R}^{\left(n_{y} q-r\right) \times\left(n_{y} q-r\right)}$ (where $\sigma_{1} \geq$ $\left.\sigma_{2} \geq \cdots \geq \sigma_{n}\right)$ represent the singular values, and where $\hat{\mathbf{U}}_{\mathbf{s}}$, $\hat{\mathbf{U}}_{\mathbf{o}}, \hat{\mathbf{V}}_{\mathbf{s}}$ and $\hat{\mathbf{V}}_{\mathbf{o}}$ are of appropriate dimensions.

It is shown that if $q \geq r$, then $\hat{\mathbf{U}}_{\mathbf{s}}=\hat{\mathbf{O}}=\mathbf{O S}\left(\mathbf{S} \in \mathbb{R}^{r \times r}\right)$ where $\hat{\mathbf{O}}$ is the extended observability matrix of the estimated $r$-th order state-space representation $\left(I_{r}, \hat{\mathbf{A}}, \hat{\mathbf{B}}, \hat{\mathbf{C}}, \hat{\mathbf{D}}\right)$

\footnotetext{
${ }^{14}$ Note that to avoid complex arithmetic, one should consider the real representation of $G=[\mathbf{R e}(\mathbf{G}) \quad \operatorname{Im}(\mathbf{G})], W=[\mathbf{R e}(\mathbf{W}) \quad \mathbf{I m}(\mathbf{W})]$ and $X=\left[\begin{array}{ll}\boldsymbol{R e}\left(\mathbf{X}^{\mathbf{c}}\right) & \left.\mathbf{I m}\left(\mathbf{X}^{\mathbf{c}}\right)\right]\end{array}\right.$
} 
is equivalent to $\left(I_{n}, \mathbf{A}, \mathbf{B}, \mathbf{C}, \mathbf{D}\right)$ with $\hat{\mathbf{A}}=\mathbf{S}^{-1} \mathbf{A S}$ and $\hat{\mathbf{C}}=$ CS. It is well-known that if the frequency-domain data are generated by an LTI system of order $r$, a clear drop appears at the $(r+1)^{t h}$ singular value of $R_{22}$ (22), then the choice of the order is obvious. If data are obtained from experimental tests, this cut off is not necessarily clear. However this decomposition can be seen as tuning parameter to obtain a satisfactory complexity / accuracy trade-off. In brief, as in the Loewner framework, thanks to the SVD decomposition the main dynamic behaviour of the system can be extracted using the singular value drop. Consequently the system order $r$ can be determined by the highest singular values of the $R_{22}$ block represented by $\hat{\Sigma}_{s}$. Thus,

$$
\hat{\mathbf{A}}=\varphi^{\dagger} b \text { and } \hat{\mathbf{C}}=J_{3} \hat{\mathbf{U}}_{\mathbf{s}},
$$

where $\varphi=J_{1} \hat{\mathbf{U}}_{\mathbf{s}}, b=J_{2} \hat{\mathbf{U}}_{\mathbf{s}}, M^{\dagger}=\left(M^{T} M\right)^{-1} M^{T}$ and

$$
\begin{aligned}
& J_{1}=\left[\begin{array}{ll}
I_{(q-1) n_{y}} & 0_{(q-1) n_{y} \times n_{y}}
\end{array}\right] \\
& J_{2}=\left[\begin{array}{ll}
0_{(q-1) n_{y} \times n_{y}} & I_{(q-1) n_{y}}
\end{array}\right] \\
& J_{3}=\left[\begin{array}{ll}
I_{n_{y}} & 0_{n_{y} \times(q-1) n_{y}}
\end{array}\right]
\end{aligned}
$$

and where

$$
J_{1} \hat{U}_{s}=\left[\begin{array}{c}
\hat{\mathbf{C}} \\
\hat{\mathbf{C}} \hat{\mathbf{A}} \\
\vdots \\
\hat{\mathbf{C}} \hat{\mathbf{A}}^{q-2}
\end{array}\right] \text { and } J_{2} \hat{U}_{s}=\left[\begin{array}{c}
\hat{\mathbf{C}} \hat{\mathbf{A}} \\
\hat{\mathbf{C}} \hat{\mathbf{A}}^{2} \\
\vdots \\
\hat{\mathbf{C}} \hat{\mathbf{A}}^{q-1}
\end{array}\right]
$$

Let us assume that $\hat{\mathbf{A}}$ and $\hat{\mathbf{C}}$ are now known, the transfer function became affine in $\hat{\mathbf{B}}$ and $\hat{\mathbf{D}}$ and can consequently be obtained by solving the following standard convex optimization problem:

$$
\{\hat{\mathbf{B}}, \hat{\mathbf{D}}\}=\arg \underset{\substack{\hat{\mathbf{B}} \in \mathbb{R}^{n \times n_{u}} \\ \hat{\mathbf{D}} \in \mathbb{R}^{n_{y} \times n_{u}}}}{ } \sum_{i=1}^{N}\left\|\boldsymbol{\Phi}_{i}-\hat{\mathbf{H}}\left(\omega_{i}, \hat{\mathbf{B}}, \hat{\mathbf{D}}\right)\right\|_{F}^{2} .
$$

where $\hat{\mathbf{H}}\left(\omega_{i}, \hat{\mathbf{B}}, \hat{\mathbf{D}}\right)=\hat{\mathbf{C}}\left(e^{\imath \omega_{i}} I_{r}-\hat{\mathbf{A}}\right)^{-1} \hat{\mathbf{B}}+\hat{\mathbf{D}}$. In addition it is shown that in the noise-free case, $\left\|\hat{\mathbf{H}}^{N}-\mathbf{H}\right\|_{\mathcal{H}_{\infty}}=0$, if $\mathbf{H}$ is a stable system of order $r$ and $N \geq r+q$ and $q \geq r$ are satisfied. Besides, this approach is consistent in the noisy case, i.e. $\lim _{N \rightarrow \infty}\left\|\hat{\mathbf{H}}^{N}-\mathbf{H}\right\|_{\mathcal{H}_{\infty}}=0$ with $q \geq r$, if $\mathbf{H}$ is a stable system of order $r$ and the noise affecting the data is a zero mean complex random variable and has bounded fourth-order moments (details are given in [27]).

Preliminary results on LMI regions for LTI systems. Before exposing the proposed algorithm, let us now recall some preliminary results on LMI regions which has initially been used in the context of controller synthesis [33], [34].

Definition 1 (LMI region): A subset $\mathcal{D}$ of the complex plane is called LMI region if there exists a symmetric matrix $\mathbf{P}=$ $\mathbf{P}^{T} \in \mathbb{R}^{n \times n}$ and a matrix $\mathbf{Q} \in \mathbb{R}^{n \times n}$ such that

$$
\mathcal{D}=\left\{z \in \mathbb{C} \mid f_{D}(z)=\mathbf{P}+\mathbf{Q} z+\mathbf{Q}^{T} \bar{z}<0\right\} .
$$

Let us notice that the characteristic function $f_{D}(z)=$ $\mathbf{P}+\mathbf{Q} z+\mathbf{Q}^{T} \bar{z}$ is a $n \times n$ Hermitian matrix. Moreover, the LMI regions are symmetric with respect to the real axis ${ }^{15}$. Without lack of generalities, inequalities (26)-(30) provide a set of characteristic functions provided as LMI regions, namely left half complex plane (26), Ellipse of centre $c_{0}$ with half-length axis $a$ and $b$ (27), Vertical strip between $\alpha_{1}$ and $\alpha_{2}$ (28), Horizontal strip between $\pm \beta$ (29), Conic sector of tip $c_{0}$ and angle $\theta$ (30).

Remark 4 (About continuous and discrete stability regions): The complex domain setting holds for both continuous and discrete systems. In the present context the discrete-domain is used, therefore the stability constraint is obtained by imposing $|z|<1$, i.e. all eigenvalues within the unit circle (by applying $a=b=1$ and $c_{0}=0$ to the ellipse characteristic function (27)). The equivalent constraint for continuous systems is then obtained by applying the standard transformation $z=e^{s T_{e}}$, where $s$ is the Laplace variable and $T_{e}$, the sampling period.

Remark 5 (Convex set intersection): The convexity property in preserved by the intersection operation on convex sets. Consequently the final subset is convex too, and, given two LMI regions $\mathcal{D}_{1}$ and $\mathcal{D}_{2}$ described by the characteristic function $f_{\mathcal{D}_{1}}$ and $f_{\mathcal{D}_{2}}$ respectively, the characteristic function $f_{\mathcal{D}}$ of $\mathcal{D}=\mathcal{D}_{1} \cap \mathcal{D}_{2}$ is simply recast as $f_{\mathcal{D}}=\operatorname{diag}\left(f_{\mathcal{D}_{1}}, f_{\mathcal{D}_{2}}\right)$.

Consequently, the formulation of poles placement objectives within LMI regions, is given by the following Theorem 2 .

Theorem 2 (LMI regions [33], [34]): Let us consider a dynamical system as in (17), $\mathbf{P}=\mathbf{P}^{T} \in \mathbb{R}^{n \times n}, \mathbf{Q} \in \mathbb{R}^{n \times n}$, then $\mathbf{A}$ has all its eigenvalues in the $\mathbf{L M I}$ regions $\mathcal{D}$ defined by the characteristic function $f_{D}(z)$ :

$$
\mathcal{D}=\left\{z \in \mathbb{C} \mid f_{D}(z)=\mathbf{P}+\mathbf{Q} z+\mathbf{Q}^{T} \bar{z}<0\right\},
$$

if and only if there exists a symmetric $\Psi=\Psi^{T}>0$ such that:

$$
\mathbf{P} \otimes \mathbf{\Psi}+\mathbf{Q} \otimes \mathbf{A} \boldsymbol{\Psi}+\mathbf{Q}^{T} \otimes(\mathbf{A} \boldsymbol{\Psi})^{T}<0 .
$$

Subspace approach under LMI regions constraints. As rooted on the above preliminary results, we are now ready to state the main result of this section, namely, the subspace approach with LMI constraints, allowing to impose the identified model poles placement.

Proposition 1 (Subspace with $\mathbf{L M I}$ regions constraints): Given $\mathbf{P}=\mathbf{P}^{T} \in \mathbb{R}^{r \times r}$ and $\mathbf{Q} \in \mathbb{R}^{r \times r}$ characterizing the LMI region $\mathcal{D}$ defined as

$$
\mathcal{D}=\left\{z \in \mathbb{C} \mid f_{D}(z)=\mathbf{P}+\mathbf{Q} z+\mathbf{Q}^{T} \bar{z}<0\right\} .
$$

The eigenvalues of $\hat{\mathbf{A}}=\varphi^{\dagger} b \in \mathbb{R}^{r \times r}$ lies in $\mathcal{D}$ if and only if the following problem:

$$
\min _{\tilde{\mathbf{A}}, \boldsymbol{\Psi}, \beta} \quad \beta
$$

subject to (35), (36), (37)

$$
\begin{aligned}
& \mathbf{P} \otimes \mathbf{\Psi}+\mathbf{Q} \otimes \tilde{\mathbf{A}}+\mathbf{Q}^{T} \otimes \tilde{\mathbf{A}}^{T}<0 \\
& \left(\begin{array}{cc}
I_{r} & (\varphi \tilde{\mathbf{A}}-b \boldsymbol{\Psi}) \\
(\varphi \tilde{\mathbf{A}}-b \boldsymbol{\Psi})^{T} & \beta I_{r}
\end{array}\right)>0
\end{aligned}
$$

${ }^{15}$ The eigenvalues of a dynamical system $\dot{x}(t)=A x(t)$ lies in $\mathbf{L M I}$ regions defined by $\mathcal{D}$ if these poles satisfied the characteristic function $f_{\mathcal{D}}$. 


$$
\begin{aligned}
\operatorname{Re}(z)<0 & \Leftrightarrow z+\bar{z}<0 \\
\left(\frac{\operatorname{Re}(z)-c_{0}}{a}\right)^{2}+\left(\frac{\operatorname{Im}(z)}{b}\right)^{2}<1 & \Leftrightarrow\left(\begin{array}{cc}
-1 & -c_{0} / a+z(1 / 2 a-1 / 2 b)+ \\
\bar{z}(1 / 2 a-1 / 2 b) \\
-c_{0} / a+\bar{z}(1 / 2 a+1 / 2 b)+
\end{array}\right. \\
z(1 / 2 a-1 / 2 b) & -r \\
\alpha_{1}<\mathbf{R e}(z)<\alpha_{2} & \Leftrightarrow\left(\begin{array}{cc}
z+\bar{z}-2 \alpha_{2} & 0 \\
0 & z+\bar{z}+2 \alpha_{1}
\end{array}\right)<0 \\
|\operatorname{Im}(z)|<\beta & \Leftrightarrow\left(\begin{array}{cc}
-2 \beta & z-\bar{z} \\
\bar{z}-z & -2 \beta
\end{array}\right)<0 \\
\left(\operatorname{Re}(z)-c_{0}\right) \tan \theta<-|\operatorname{Im}(z)| & \Leftrightarrow\left(\begin{array}{cc}
\left(-2 c_{0}+z+\bar{z}\right) \sin \theta & (z-\bar{z}) \cos \theta \\
(z-\bar{z}) \cos \theta & \left(-2 c_{0}+z+\bar{z}\right) \sin \theta
\end{array}\right)<0
\end{aligned}
$$

where $\tilde{\mathbf{A}}=\hat{\mathbf{A}} \boldsymbol{\Psi} \in \mathbb{R}^{r \times r}$ and $\boldsymbol{\Psi}=\boldsymbol{\Psi}^{T} \in \mathbb{R}^{r \times r}$ and $\beta \in \mathbb{R}$, has a solution.

Proof: Thanks to Theorem 2, eigenvalues of $\hat{\mathbf{A}}$ are located in the $\mathbf{L M I}$ region $\mathcal{D}$ if $\boldsymbol{\Psi}=\boldsymbol{\Psi}^{T}>0$ exists such that:

$$
\begin{aligned}
\mathbf{P} \otimes \boldsymbol{\Psi}+\mathbf{Q} \otimes \hat{\mathbf{A}} \boldsymbol{\Psi}+\mathbf{Q}^{T} \otimes(\hat{\mathbf{A}} \boldsymbol{\Psi})^{T} & <0 \\
\Leftrightarrow \mathbf{P} \otimes \boldsymbol{\Psi}+\mathbf{Q} \otimes \tilde{\mathbf{A}}+\mathbf{Q}^{T} \otimes \tilde{\mathbf{A}}^{T} & <0,
\end{aligned}
$$

where $\tilde{A}=\hat{A} \Psi$. Besides, let $J_{1} \hat{U}_{s} \hat{\mathbf{A}}=J_{2} \hat{U}_{s} \Leftrightarrow \varphi \hat{\mathbf{A}}=b$ with $\varphi=J_{1} \hat{U}_{s}$ and $b=J_{2} \hat{U}_{s}$. Since $\varphi \hat{\mathbf{A}}=b$ then $\varphi \hat{\mathbf{A}} \boldsymbol{\Psi}=b \boldsymbol{\Psi} \Leftrightarrow$ $\varphi \tilde{\mathbf{A}}=b \tilde{\Psi}$ with $\tilde{\mathbf{A}}=\tilde{\mathbf{A}} \boldsymbol{\Psi}$. Finding $\tilde{\mathbf{A}}$ is equivalent to solve $\min \|\varphi \tilde{\mathbf{A}}-b \boldsymbol{\Psi}\|_{2}^{2}$. Consequently, one can write:

$$
\min \|\varphi \tilde{\mathbf{A}}-b \Psi\|_{2}^{2} \Leftrightarrow \quad \begin{gathered}
\min _{\beta \in \mathbb{R}} \beta>0 \\
(\varphi \tilde{\mathbf{A}}-b \Psi)^{T}(\varphi \tilde{\mathbf{A}}-b \Psi)<\beta I_{r} .
\end{gathered}
$$

Then, a quadratic cost function has been transformed into a linear cost function under quadratic constraints. Consequently, by invoking the Schur lemma, one obtains:

$$
\Leftrightarrow\left(\begin{array}{cc}
(\varphi \tilde{\mathbf{A}}-b \boldsymbol{\Psi})^{T}(\varphi \tilde{\mathbf{A}}-b \boldsymbol{\Psi}) & <\beta I_{r} \\
I_{r} & (\varphi \tilde{\mathbf{A}}-b \boldsymbol{\Psi}) \\
(\varphi \tilde{\mathbf{A}}-b \boldsymbol{\Psi})^{T} & \beta I_{r}
\end{array}\right)>0
$$

where matrix inequalities clearly are affine in decision variables $\beta, \tilde{\mathbf{A}}$ and $\boldsymbol{\Psi}$.

A numerical procedure to obtain a dynamical model satisfying the eigenvalue oriented LMI constraint is sketched in what follows (see Algorithm 1).

Remark 6 (Handling noisy data): In the noisy data context, to preserve consistency, a weighting matrix $\mathbf{N}$ is used based on the noise relative variance. This matrix must be a priori known. The evaluation is strictly equivalent and can be included in our optimization problem as follows:

$$
\begin{aligned}
& \hat{\mathbf{A}}=\left(J_{1} \mathbf{N} \hat{\mathbf{U}}_{1}\right)^{\dagger} J_{2} \mathbf{N} \hat{\mathbf{U}}_{\mathbf{1}} \Leftrightarrow \hat{\mathbf{A}}=\varphi^{\dagger} b \\
& \hat{\mathbf{C}}=J_{3} \mathbf{N U}_{\mathbf{1}}
\end{aligned}
$$

where $\varphi=J_{1} \mathbf{N} \hat{\mathbf{U}}_{\mathbf{1}}$ and $b=J_{2} \mathbf{N} \hat{\mathbf{U}}_{\mathbf{1}}$.

Remark 7 (LMI constraints): If no constraints is considered, the LMI least-square solution is exactly equivalent to the baseline subspace approach. Moreover, one should note that in practice, the consistency of the problem is not affected by imposing poles placement regions if these constraints are relevant with the system to identify [27].

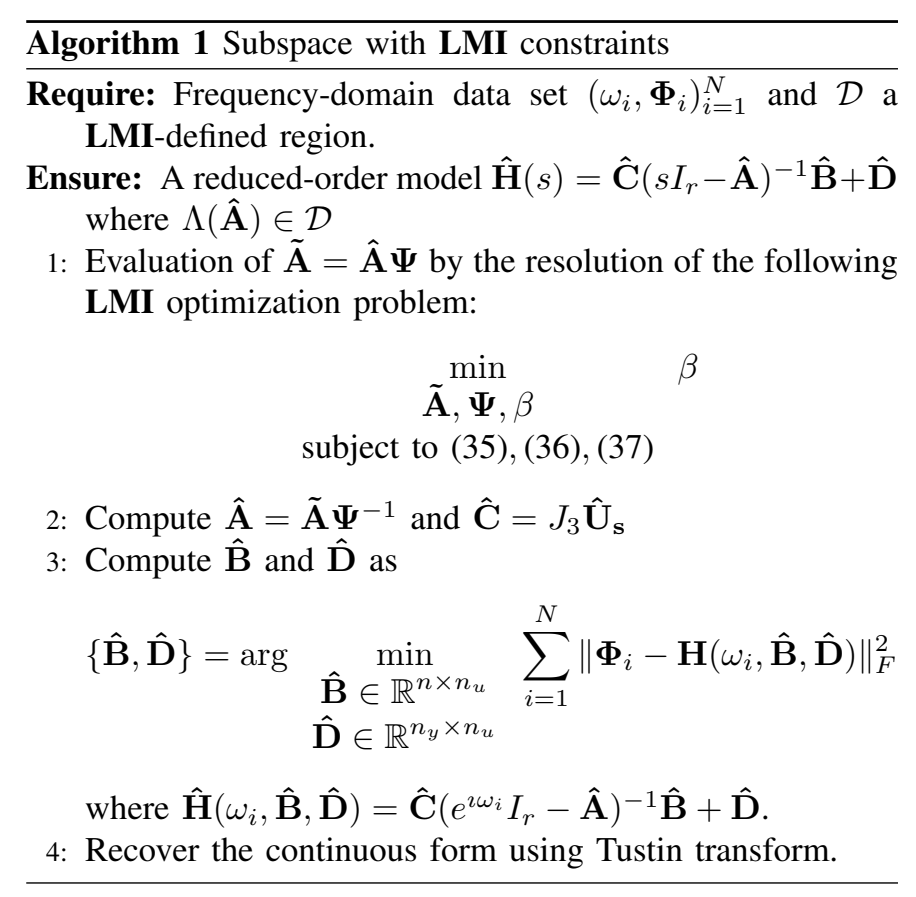

Application to the WT data. For the considered application, Algorithm 1 has been involved with $N=292$ sampled data points, $n_{y}=3$ outputs (acceleration at stations \#4, \#12 and \#15) and $n_{u}=2$ inputs (wind gust and actuator) and a sample frequency $f_{s}=4096 \mathrm{~Hz}^{16}$. When applying Algorithm 1, the considered LMI region is an ellipse defined by the constraint (27) with $a=1, b=0.92$ and $c_{0}=0$. This ellipse leads to impose the stability since this LMI region is included in the unitary disk. Furthermore, the half-length axis $b=0.92$ avoids to under-estimate the modes damping and to delete possible weakly damped poles which can appear and which are not physical.

First, the vertical acceleration output corresponding to station \#15 is illustrated on Figure 6, with $r=20$ (on the same configurations as the one shown on Figure 5 for the Loewner approach). Comparing with Figure 5, this figure illustrates the very good performance of the subspace approach.

\footnotetext{
${ }^{16} \mathrm{As}$ the sampling frequency $\left(f_{s}=4096 \mathrm{~Hz}\right)$ is widely superior to the main system dynamics, the continuous and discrete frequency domain responses are strictly equal for frequencies inferior to $100 \mathrm{~Hz}$.
} 


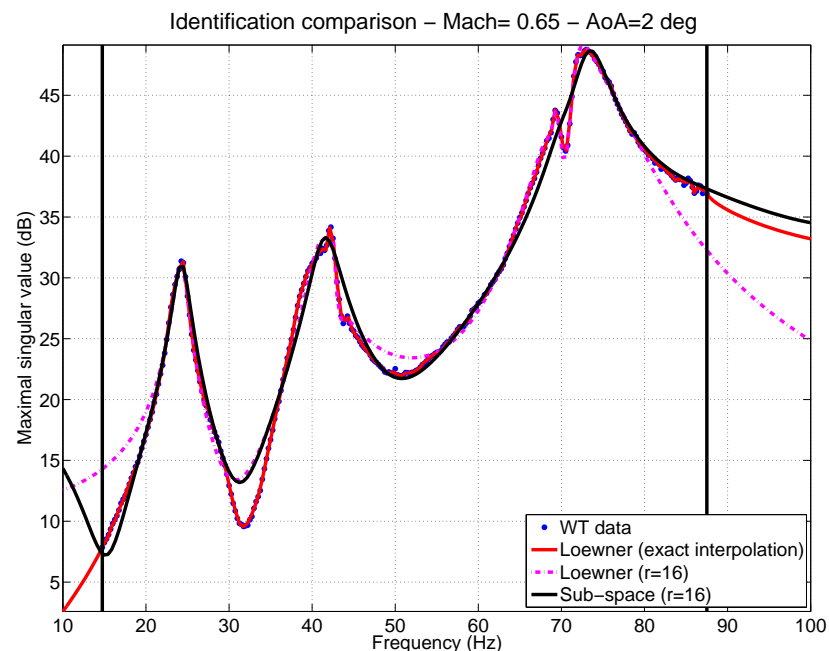

Fig. 7. Comparison of the maximal singular values response (gain) of the wing vertical accelerations at station \#4, \#12 and \#15 obtained in WT experiments (dotted blue) with models obtained via Loewner approach (exact in red, approximate in pink dashed) and subspace-based with LMI constraints (solid black) with $r=16$ (dash-dotted magenta) interpolation. Vertical lines materialize the limit of the considered data set $\left(\omega_{i}, \boldsymbol{\Phi}_{i}\right)_{i=1}^{N}$.

Then, Figure 7 compares the maximal singular values of the MIMO models obtained with either the exact and approximate Loewner approach and the subspace with LMI constraint one, for $r=16$ (order of the model used in the control design Section III). It emphasizes the fact that the subspace with LMI constraint obtained model (black solid thin) well matches the measurement points (blue points).

In addition, Figures 8 and 9 present the maximal singular value responses and eigenvalues of both the baseline subspace approach and the LMI-based subspace one. On Figure 8, the identified models for both approaches are illustrated. It is notable that both methods lead to a good approximation of the frequency data. More interestingly, Figure 9 shows the corresponding eigenvalues in the discrete domain (left) and the equivalent in the continuous one (right). First, one should note that the subspace approach without LMI constraint might lead to models with unstable modes (here two unstable modes). Besides, the proposed approach leads to models where the modes are stable and are gathered in the defined LMI region constraints (materialized by the solid blue line).

\section{ACTIVE CLOSED-LOOP LOAD ALLEVIATION CONTROL DESIGN AND DEMONSTRATION}

\section{A. Control set-up description}

Once, at each AoA and Mach number configurations, a model $\hat{\mathbf{H}}^{(i)}\left(i=1, \ldots, n_{s}\right.$, where $n_{s}$ is the number of models/configurations) have been identified, the second contribution of this work is to design an active closed-loop feedback controller aiming at attenuating the first amplification peak (heave structural motion illustrated on Figure 1) through the actuation of the movable surface. Before describing the load controller design approach, two main remarks should be kept in mind for the design: (i) first, the controller should be

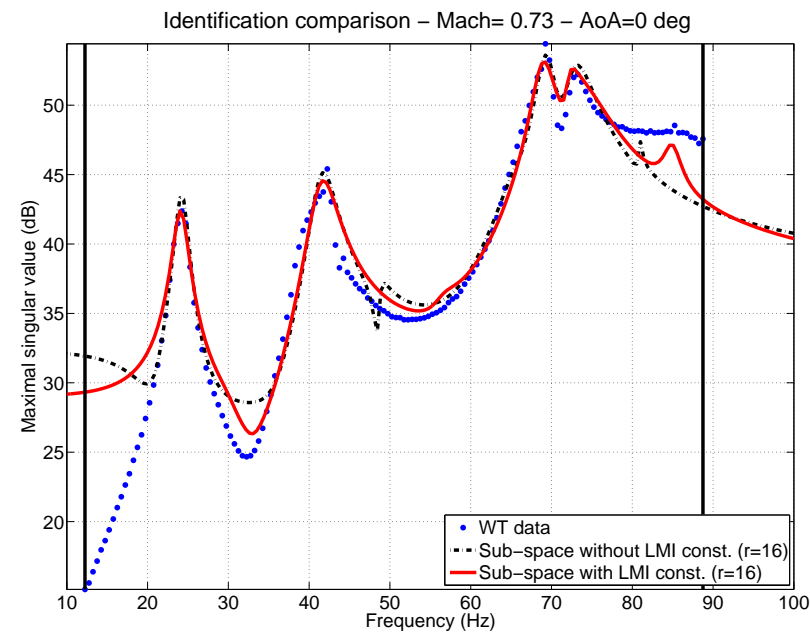

Fig. 8. Second approach (comparison): maximal singular value response (gain) of the vertical accelerations of the wing at stations \#4, \#12 and \#15 obtained in WT experiments (dotted blue), with model obtained via subspacebased without LMI constraints (dash-dotted black) and with LMI constraints (solid red), with $r=16$. Vertical lines materialize the limit of the considered data set $\left(\omega_{i}, \boldsymbol{\Phi}_{i}\right)_{i=1}^{N}$

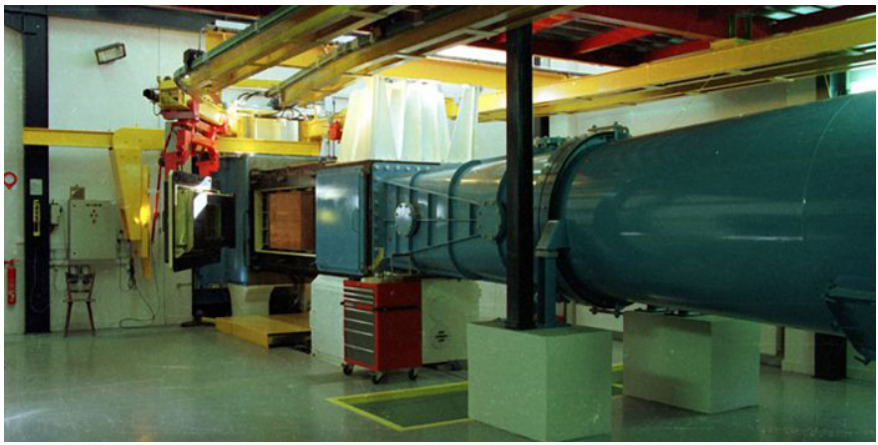

Fig. 10. Photo of the ONERA research wind tunnel facility (flow going from the back to the front).

easy to implement on real-time device ${ }^{17}$ (Figure 10) (ii) and second, it should be robust to all Mach number and AoA variations ${ }^{18}$.

With reference to Figure 2, the control signal $u(t)$ is the control surface deflection and the measurement vector $\mathbf{y}(t)=\left[\ddot{z}_{\# 4}(t) \ddot{z}_{\# 15}(t)\right]$ used for the control is composed of the acceleration sensors at station \#4 and \#15.

Remark 8 (Considerations about the selected models): As mentioned in the introduction, for the control design step, the models obtained using the subspace with LMI constraint approach have been selected (with dimension $r=16$ ). The models of high order ( $n=292$ ) obtained by the exact Loewner approach has been used for validation purpose. Indeed, while the latter catches all the dynamics of the frequency response (but may result in instability), which are well appropriated for frequency domain validation, the former are more tailored

\footnotetext{
${ }^{17}$ Since wind tunnel experiments are time consuming and expensive, the ability in easily implementing and re-tune the control law is primordial.

${ }^{18}$ Indeed, for the considered application, one is interested in a single robust linear time invariant controller instead of a parameter varying one, which would be addressed in a future step of the study.
} 

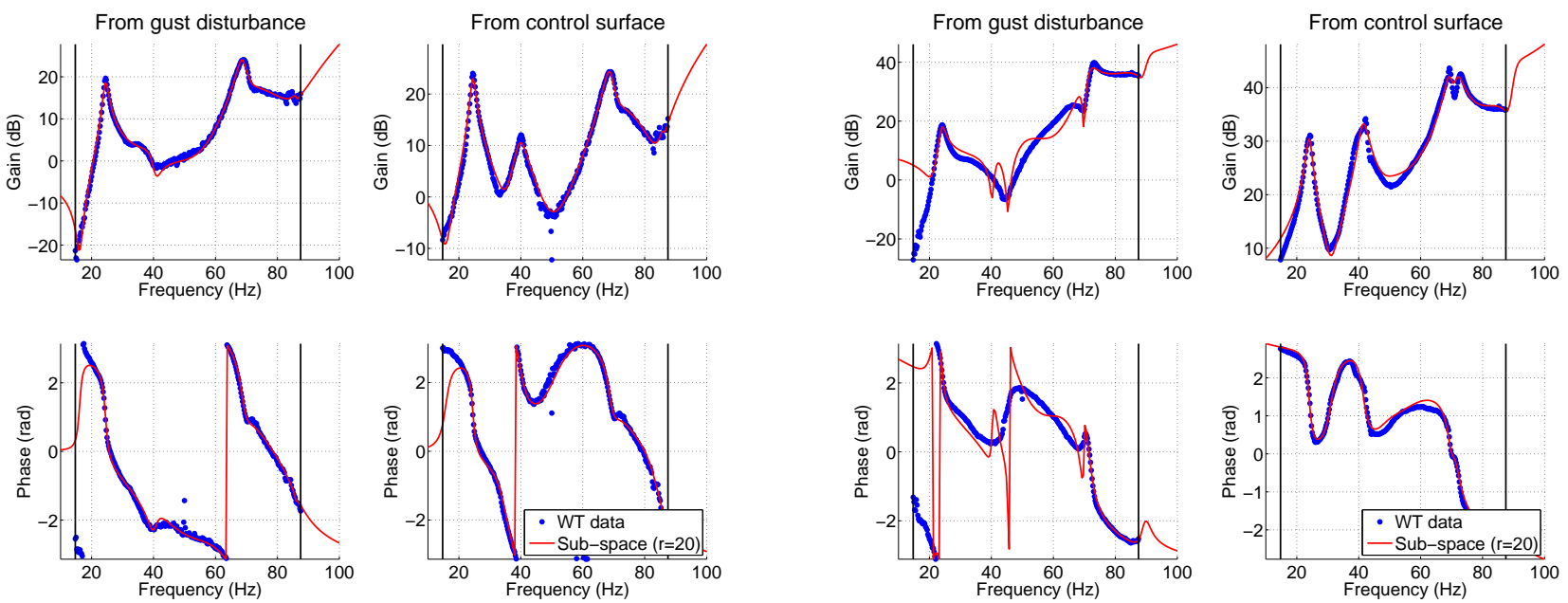

Fig. 6. Second approach: comparison of the frequency response (gain and phase) of the vertical acceleration of the wing at station \#15 obtained in WT experiments (dotted blue) with models obtained via subspace-based with LMI constraints (solid red) and reduced with $r=20$ (dash-dotted magenta) interpolation. Left: Mach number 0.30 and AoA 0 degree. Right: Mach number 0.73 and AoA 0 degree. Vertical lines materialize the limit of the considered data set $\left(\omega_{i}, \boldsymbol{\Phi}_{i}\right)_{i=1}^{N}$.
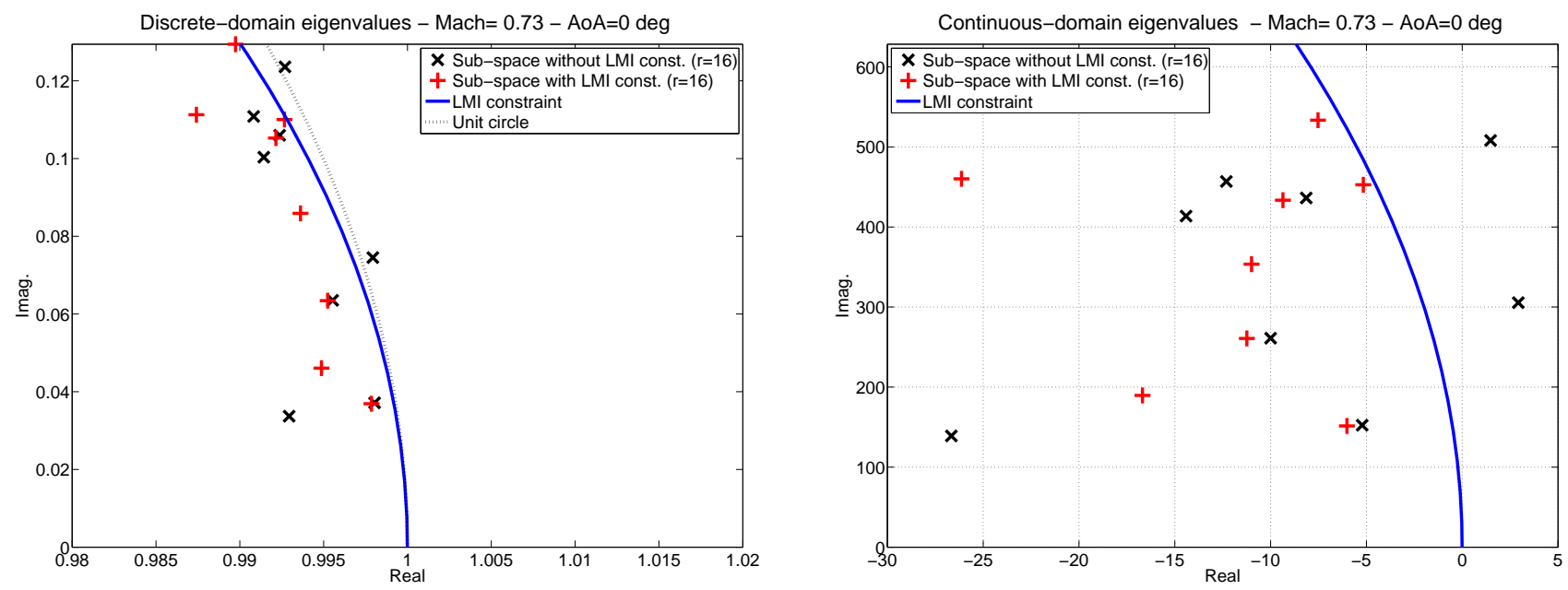

Fig. 9. Second approach (comparison): eigenvalues of the obtained models with the subspace approach, with $(+)$ and without $(\times)$ LMI constraints, with $r=16$. Left: discrete-domain models eigenvalues. Right: equivalent continuous-domain eigenvalues. The blue lines represent the equivalent LMI domain constraints. Left: in the discrete-domain (evaluation of (27) with $a=1, b=0.92$ and $c_{0}=0$ ). Right: equivalence in the continuous-domain by the transformation $s=\frac{1}{T e} \ln (z)$.

for controller design since they preserve model stability and constrain badly damped modes ${ }^{19}$. From a practical point of view it is interesting to notice that both models are used for different purposes ${ }^{20}$.

\section{B. Robust controller design}

According to the above preliminary objective, a controller is synthesized using a robust approach in order to obtain $\mathbf{K}^{\star}$,

\footnotetext{
${ }^{19}$ Indeed, avoiding artificial unstable modes alleviates the control optimization procedure to stabilize irrelevant and unrealistic modes.

${ }^{20}$ The subspace approach, using $\mathbf{L M I}$ is quite sensitive to the dimension of the seek model and models should be kept quite small (i.e. in practice, $r<20$ ) but can guarantee stability. On the other side, the Loewner models are not sensitive to the approximation order $r \gg 100$ but might result in unstable models. Since it is not the scope of the paper, no further analysis is given here.
}

an LTI controller that is both robust to the Mach number and AoA variations. To address this objective, the synthesis problem is recast as a multi-channel $\mathcal{H}_{\infty}$-norm minimization one $^{21}$. Note that the robust consideration justifies the use of an $\mathcal{H}_{\infty}$ approach instead of the $\mathcal{H}_{2}$ one, which might appear more appropriate in view of the root mean square minimization. Mathematically, the objective is to find the optimal controller

\footnotetext{
${ }^{21}$ Without lack of generalities, by designing adequate weighting filters to generate the generalized plant for transfer minimization, the $\mathcal{H}_{2}$ objective can be - in some sense - recast as an $\mathcal{H}_{\infty}$ one. As it will be made clearer in the following, this formulation also allows to handle additional constraints on the controller properties.
} 
$\mathbf{K}^{\star}$ such that,

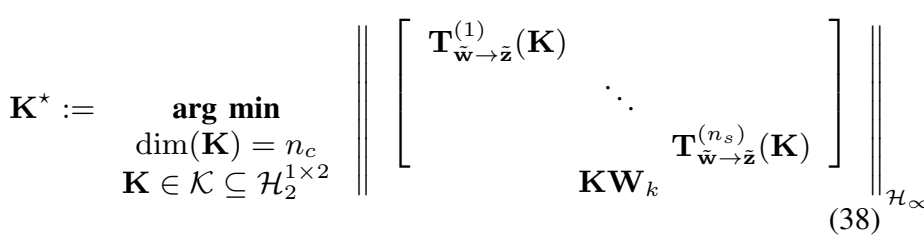

where $\mathcal{K}$ describes the considered controller family set (see hereafter for more details), $n_{c} \in \mathbb{N}^{*}$ is the controller order, and $\mathbf{T}_{\tilde{\mathbf{w}} \rightarrow \tilde{\mathbf{z}}}^{(i)}(\mathbf{K})$ (for $i=1, \ldots, n_{s}$ ) represents the $\mathbf{K}$ dependent performance transfer function from $\tilde{\mathbf{w}}$ to $\tilde{\mathbf{z}}$ (see hereafter and Figure 11). Finally, $\mathbf{W}_{k}$ is an additional weighting function used (i) first to enforce transfer $\mathbf{K W}_{k}$ to be stable, and thus $\mathbf{K}$ to be stable ( $\mathbf{W}_{k}$ being stable), and (ii) second, to shape $\mathbf{K}$ roll-off behaviour. More specifically, we have

$$
\mathbf{W}_{k}=G_{k} \frac{s^{2} / w_{r o}^{2}}{s^{2} /\left(10^{3} w_{r o}\right)^{2}+s 1.4 /\left(10^{3} w_{r o}\right)+1}
$$

where $G_{k}=10^{-2}$ and $w_{r o}=2 \pi 30$ are the filter low frequency gain and roll-off cutting frequency, respectively.

1) Considerations about $\mathcal{K}$ : With reference to (38), $\mathcal{K}$ is the set of strictly stable rational transfer functions of order $n_{c}$. Indeed $\mathbf{K} \in \mathcal{K} \subseteq \mathcal{H}_{2}^{1 \times 2}$ is a structure-defined set of rational transfer functions in $\mathbb{C}^{1 \times 2}$ ( 2 measurements, 1 control signal $)$ whose structure is defined as

$$
\mathbf{K}(s)=\mathbf{C}_{K}\left(s I_{n_{c}}-\mathbf{A}_{K}\right)^{-1} \mathbf{B}_{K},
$$

where $\mathbf{A}_{K} \in \mathbb{R}^{n_{c} \times n_{c}}, \mathbf{B}_{K} \in \mathbb{R}^{n_{c} \times 2}$ and $\mathbf{C}_{K} \in \mathbb{R}^{n_{c} \times 1}$ are tuning parameters to be determined. More specifically, the optimal controller $\mathbf{K}^{\star} \in \mathcal{K}$ will have the structure presented in (40). Obviously, the main interest in structuring the control law as in (40) is to find a law that is both simple and performs appropriately to attenuate the targeted peak. Moreover, thanks to constraint (39), the obtained controller should have a roll-off behaviour after $w_{r o}$. Designing such a controller can be easily achieved thanks to recent advances in dedicated numerical tools. In the considered case, the approach developed in [20] has been invoked.

2) Considerations about $\mathbf{T}_{\tilde{\mathbf{w}} \rightarrow \tilde{\mathbf{z}}}^{(i)}(\mathbf{K})$ : With reference to (38), the transfer functions depending on $\mathbf{K}$, describing the the performance channels are defined as $\left(i=1, \ldots, n_{s}\right)$ :

$$
\mathbf{T}_{\tilde{\mathbf{w}} \rightarrow \tilde{\mathbf{z}}}^{(i)}(\mathbf{K})=\mathbf{W}_{I} \mathcal{F}_{l}\left(\hat{\mathbf{H}}^{(i)}, \mathbf{K}\right) \mathbf{W}_{O}
$$

where,

$$
\mathbf{W}_{O}(s)=\left[\begin{array}{cc}
\mathbf{W}_{p}(s) & 0 \\
0 & \mathbf{W}_{u}(s)
\end{array}\right] \text { and } \mathbf{W}_{I}(s)=1
$$

and where (see also Figure 11),

- The performance channel is characterized by $\mathbf{W}_{p}(s)$, defined as

$$
\mathbf{W}_{p}(s)=\frac{G_{\infty}\left(s / 10 w_{p}+1\right)}{s / w_{p}+1} I_{3},
$$

where $G_{\infty}$ is the frequency-limited $\mathcal{H}_{\infty}$-norm (evaluated over $\Omega$ ) of the performance transfer from $\tilde{\mathbf{w}}$ to $\tilde{\mathbf{z}}$, and $w_{p}=2 \pi 30 \mathrm{rad} / \mathrm{s}$. This performance channel is applied on all the considered output, i.e. the accelerations at stations \#4, \#12 and \#15, with objective to attenuate the considered dimensioning heave peak.

- Then, the control input signal attenuation level, aiming at preserving eventual control saturation is given as

$$
\mathbf{W}_{u}(s)=\frac{s / w_{a c t}+1}{s / 10 w_{a c t}+1},
$$

where $w_{\text {act }}=2 \pi 30 \mathrm{rad} / \mathrm{s}$.

With reference to Figure 11, $\hat{\mathbf{H}}^{(i)}$ denotes the identified model obtained using the subspace approach described in Section II-B with order $r=16$ (see Figures 7 and 8), describing the system behaviour for varying AoA and Mach number. Figure 11 illustrates the interconnection for the $i$ th transfer.

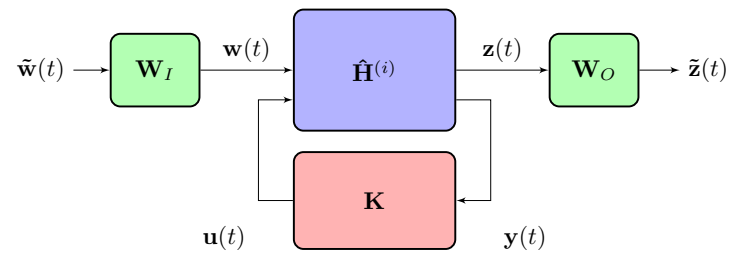

Fig. 11. Generalized control scheme of the $i$ th transfer $\mathbf{T}_{\tilde{\mathbf{w}} \rightarrow \tilde{\mathbf{z}}}^{(i)}(\mathbf{K})$.

For sake of completeness, $\mathbf{u}(t)$ and $\mathbf{y}(t)$ signals denote the control surface deflection signal $u(t)$ and the vertical accelerations at stations \#4 and \#15, respectively. Then, $\mathbf{w}(t)$ and $\mathbf{z}(t)$ are the gust disturbance input $w(t)$ and vertical accelerations output at stations \#4, \#12 and \#15 (to be attenuated), respectively. Finally, $\tilde{\mathbf{w}}(t)$ and $\tilde{\mathbf{z}}(t)$ are the filtered exogenous input and output, respectively, defining the generalized plant and input/output transfer functions to be minimized by the $\mathcal{H}_{\infty}$-norm objective.

\section{Numerical and experimental validation}

In practice, solving (38) with $n_{s}=5$ and a LTI controller $\mathbf{K}$ of order $n_{c}=8$ has been obtained using the approach described in $[20]^{22}$. The obtained controller presents a frequency behaviour consistent with the constraints given by (38), namely, $\mathbf{K}^{\star} \in \mathcal{K}$, with a low gain action in low frequencies (indeed, only the peak has to be attenuated) and roll-off in higher ones (satisfying $\mathbf{W}_{k}$ as stated in (39)) $)^{23}$. The achieved attenuation level, solution of the optimization problem (38), leads to an optimal $\mathcal{H}_{\infty}$-norm value of $0.43473(<1)$, meaning that the weighting function constraints are attenuated (especially the performance criteria $\mathbf{W}_{p}$ one).

Before moving to the implementation step, in order to assess the obtained controller performance, simulation are first performed by applying the optimal $\mathbf{K}^{\star}$ on the complete model obtained with the exact Loewner approach (Section II-A) ${ }^{24}$.

\footnotetext{
${ }^{22}$ Five configurations of open-loop systems for Mach number going from 0.30 to 0.73, and $\mathbf{A o A}$ from 0 to 2 degree.

${ }^{23}$ One should note that the low gain in low frequency is non standard in traditional control set-up where tracking and low frequency disturbances are usually treated. However, in the considered context, neither tracking nor step disturbances are considered, which justifies this low frequency attenuation behaviour.

${ }^{24}$ Note that here the exact Loewner model is used since, even is nominally unstable, it perfectly captures the frequency data over all the considered frequency range.
} 
The left frame of Figure 12 both provides open-loop and closed-loop frequency responses and performance attenuations for varying configurations from sub to transonic (varying AoA and Mach number configurations).

Once the attenuation level is numerically validated, similar experiments are performed in the WT facility and results are reported on the right frame of Figure 12. By comparing the RMS gain displayed on both (left and right) frames of Figure 12 , one can observe that they are quite close to each other. The only difference between both results is concerned with the notch-like behaviour observed on Figure 12 around $40 \mathrm{~Hz}$ at configurations of AoA of 2 degrees, which has been observed in late experiments. This discrepancy can be explained by the friction effects that slightly modified the system behaviour due to intensive use of the experimental set-up ${ }^{25}$. Nevertheless, this varying behaviour illustrates that the implemented control law is also robust with respect to the plant dynamical variations.

Remark 9 (Implementation specifications \& tested configurations): The synthesized controllers were implemented on a real time device which was comprised of several processors and input/output boards interlinked for fast internal communication and data exchange. The I/O interface was composed of a maximum of 15 analog inputs and 18 analog outputs. A dedicated computer was used for creating, compiling and implementing the control laws in the processor boards and a real-time man/machine interface was developed to monitor the signals and change control/command parameters. Synthesized in the continuous time-domain, the controllers were converted into discrete state-space models to be directly implemented. With respect to the bandwidth of the electrohydraulic actuator, a $2 \mathrm{kHz}$ sampling frequency was selected. Regarding the considered application within the WT, all the configurations used in the robust design approach have been tested, while in between configurations have not been tested for time limitations. However, extension of this work might include other tests such as a more complete design, involving a parametric / uncertain model instead of local models, as stated in (38).

In addition to the frequency-domain data collected in WT and compared with simulation (Figure 12), a time-domain gust response have also been performed in simulation only ${ }^{26}$. This time-domain simulation, which consists of a discrete gust response simulated by 1-cosine disturbance wave, is given as follows:

$$
w(t)=\frac{U_{d s}}{2} \times\left[1-\cos \left(\frac{\pi V_{T A S}}{H} t\right)\right] .
$$

This disturbance shape is similar to the one used by aircraft manufacturers to validate the load alleviation functions where $V_{T A S}=248 \mathrm{~m} / \mathrm{s}$ is the true airspeed, $U_{d s}$ the amplitude which varies from 11.8 to $19 \mathrm{~m} / \mathrm{s}$ and $H$ the scale which lies from 1.52 to $7.62 \mathrm{~m}$. Figure 13 gathers all the open-loop (dashed blue) and closed-loop (solid red) responses of the vertical acceleration at station \#15 to the wind gust disturbance (45) for

\footnotetext{
${ }^{25}$ Note that this dynamic is linked to the pitch behaviour (see Figure 2) which is, after recurrent wind tunnel experimental tests, affected by viscous friction effect.

${ }^{26}$ It was experimentally impossible to generate the same kind of test in WT due to the limited amplitude range of the gust generator.
}

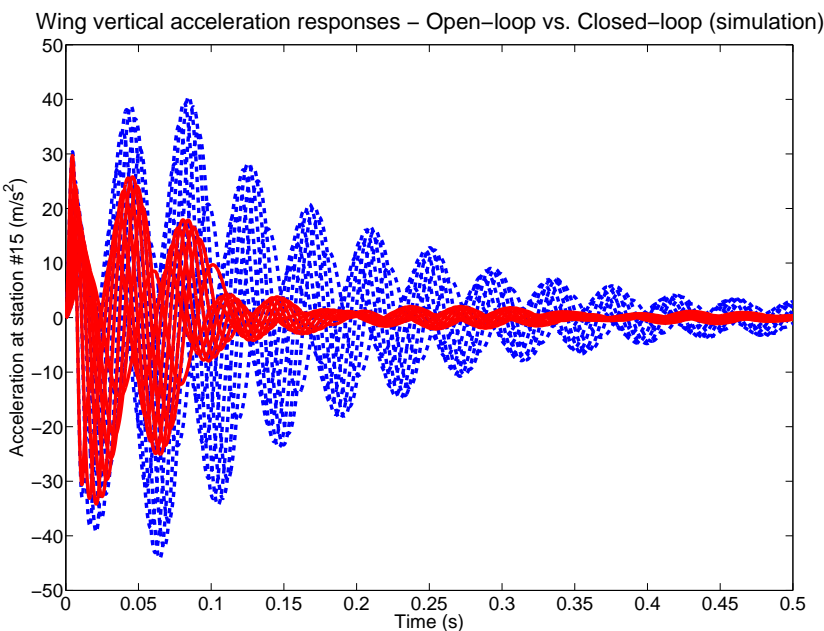

Fig. 13. Time-domain responses in open-loop (dashed blue) and closed-loop (solid red) for all the the gust configurations obtained $\left(H \in\left[\begin{array}{ll}1.52 & 7.62\end{array}\right] \mathrm{m}\right.$ and $U_{d s} \in\left[\begin{array}{ll}11.8 & 19\end{array}\right] \mathrm{m} / \mathrm{s}$ at AoA 2 degree and Mach number 0.73).

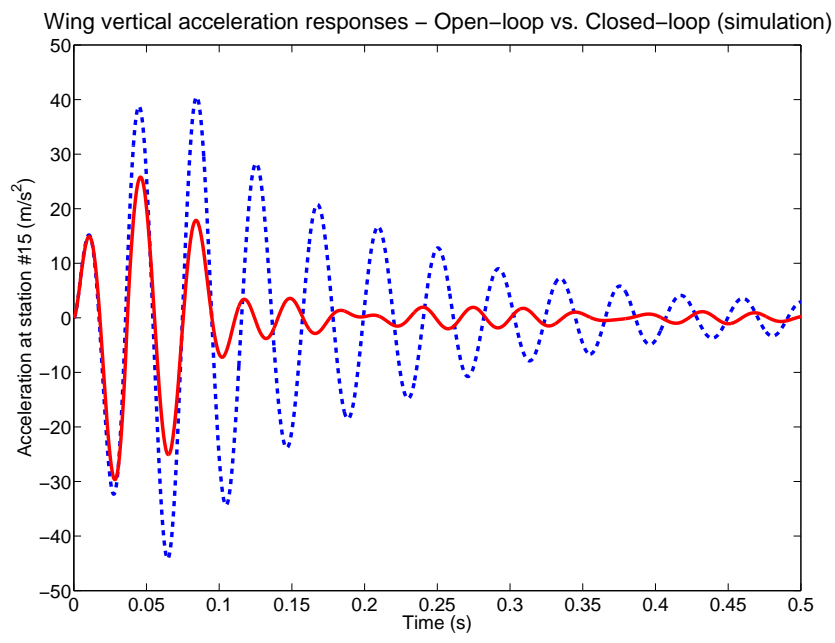

Fig. 14. Time-domain response in open-loop (dashed blue) and closed-loop (solid red) for the worst case gust configuration obtained ( $H=3.81 \mathrm{~m}$ and $U_{d s}=17.4 \mathrm{~m} / \mathrm{s}$ at AoA 2 degree and Mach number 0.73 ).

varying gust length and amplitude, derived from certification specifications.

Figure 13 clearly illustrates that the proposed algorithm attenuates the gust effect for all configurations, especially the critical ones, e.g. those where the acceleration is the larger, and which thus is dimensioning for the aircraft design. Finally, the gust effect on the so-called worst case configuration, is given on Figure 14, where the load alleviation function clearly shows a great attenuation level on the vertical structural responses.

\section{CONCLUSION AND PERSPECTIVES}

In this paper a complete illustration of the benefits brought by an active closed-loop gust load alleviation controller has been presented and demonstrated on both sub and transonic configurations. This demonstration involves many research fields, going from the manufacturing and aeroelasticity to the 

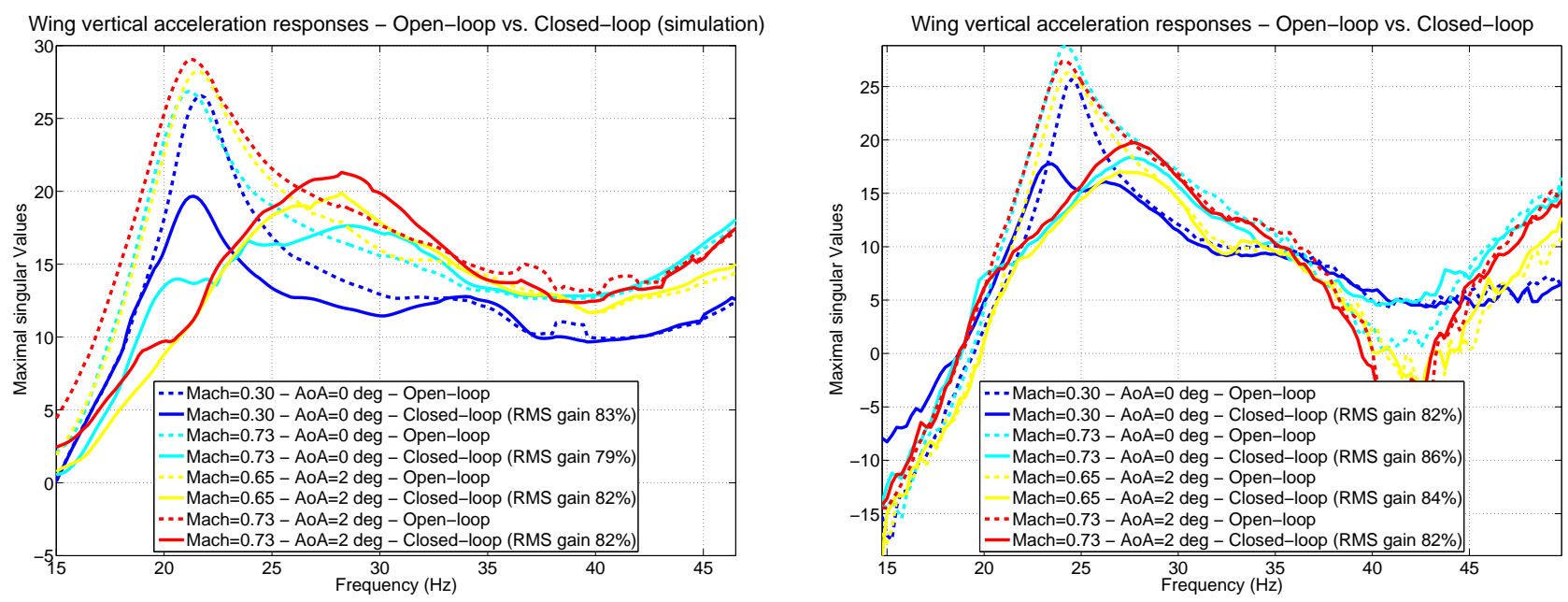

Fig. 12. Frequency responses of the wing vertical acceleration at station \#15, in response to gust - obtained in simulation (left) and WT facility (right), without (dashed) and with (solid) gust load alleviation functions. Varying colours for varying AoA and Mach number conditions. The RMS gain is also computed over the spectrum $[1550] \mathrm{Hz}$.

identification and control design. Finally, the real implementation in a research-oriented experimental set-up has been achieved.

Moreover, from a methodological point of view, two main contributions are also presented. The first one consists of the development an innovative frequency-based identification procedure with eigenvalues constraints using a subspace approach $^{27}$. The second one is the use and application of the very recently developed structured $\mathcal{H}_{\infty}$-norm minimization oriented tools to adjust a control law that can be used - as it - in the closed-loop experimental set-up of the ONERA wind tunnel facility. The overall approach provides a baseline/rationale to the gust load attenuation problem, through a model-based control methodology. More specifically, on the considered wind tunnel set-up, a RMS attenuation level of $80 \%$ (from open to closed-loop) has been achieved in between 15 and $50 \mathrm{~Hz}$, with a simple linear time invariant control law. Then, using time-domain simulations, and more specifically the worst case configuration, an attenuation level of $35 \%$ was observed, which is considerable and relevant for aeronautical applications. In view of a future application on a real aircraft, the next step is to assess the overall approach on a 3D model to increase the acceptability and reach aeronautical readiness level.

Finally, a media material is made available at http://www.onera.fr/en/news/gust-effect-control-a-europeanfirst-at-onera. It illustrates the benefit achieved by the active gust load control at $25 \mathrm{~Hz}$ (bending mode) obtained in WT experimental facility of ONERA S3Ch, in transonic configuration (Mach number 0.73), with an AoA of 0 degree. The video, recorded with a high speed camera, emphasizes the control benefit. Open-loop: from 0 to 18 seconds. Closed-loop: from 18 seconds to the end. Indeed, in the open-loop configuration, the vertical structural response of

\footnotetext{
${ }^{27}$ Which is compared to the very powerful Loewner approach, which main drawback is the lack of control on the obtained eigenvalues.
}

the wing are large, while, when the active control is activated, a clear diminution of the displacement level is observed.

\section{ACKNOWLEDGEMENT}

The research leading to these results has received funding from the European Union's Seventh Framework Program (FP7/2007-2013) for the Clean Sky Joint Technology Initiative under grant agreement CSJU-GAM-SFWA-2008-001.

\section{REFERENCES}

[1] F. Demourant and G. Ferreres, "A frequency domain identificationcontrol approach for a flexible aircraft," in Proceedings of the 2002 International Conference on Control Applications, 2002, pp. 126-131.

[2] B. Moulin and M. Karpel, "Gust load alleviation using special control surfaces," Journal of Aircraft, vol. 44, no. 1, pp. 17-25, 2007.

[3] J. Torralba, G. Puyou, and F. Demourant, "Self-scheduling multiobjective control law design for a flexible aircraft," in Guidance, Navigation, and Control and Co-located Conferences. American Institute of Aeronautics and Astronautics, August 2009.

[4] N. Aouf, B. Boulet, and R. Botez, "Robust gust load alleviation for a flexible aircraft," Canadian Aeronautics and Space Journal, vol. 46, no. 3, pp. 131-139, 2010.

[5] S. Haghighat, H. Liu, and J. Martins, "A model predictive gust load alleviation controller for a highly flexible aircraft," Journal of Guidance, Control and Dynamics, 2012.

[6] H.-G. Giesseler, M. Kopf, T. Faulwasser, P. Varutti, and R. Findeisen, "Gust load alleviation based on model predictive control," in Proceedings of the IFASD, 2012.

[7] F. Demourant and G. Ferreres, "A linear parameter-varying multiobjective control law design based on youla parametrization for a flexible blended wing body aircraft," in EUCASS Proceedings Series in Advances in AeroSpace Sciences, Array, Ed., vol. 6, 2013, pp. 729-748.

[8] J. Xu and I. Kroo, "Aircraft design with active load alleviation and natural laminar flow," Journal of Aircraft, vol. 51, no. 5, pp. 1532-1545, September 2014.

[9] M. Alama, M. Hromcikb, and T. Hanis, "Active gust load alleviation system for flexible aircraft: Mixed feedforward/feedback approach," Aerospace Science and Technology, vol. 41, pp. 122 - 133, February 2015.

[10] A. Schirrer, M. Kozek, F. Demourant, and G. Ferreres, Feedback Control Designs in Modeling and Control for a Blended Wing Body Aircraft, ser. Advances in Industrial Control, M. Kozek and A. Schirrer, Eds. Springer International Publishing, 2015. 
[11] D. Tang, P. Cizmas, and E. Dowell, "Experiments and Analysis for a gust generator in a wind tunnel," Journal of Aircraft, vol. 33, no. 1, pp. 139-148, January-February 1996.

[12] S. Ricci and A. Scotti, "Wind tunnel testing of an active controlled wing under gust excitation," in 49th AIAA/ASME/ASCE/AHS/ASC Structures, Structural Dynamics and Material Conference, Schaumburg, Illinois, USA, April 2008.

[13] W. Silva, E. Vartio, A. Shimko, R. Kvaternik, K. Eure, and R. Scott, "Development of aeroservoelastic analytical models and gust load alleviation control laws of a sensor craft wind-tunnel model using measured data," in IFASD 2007, International Forum on Aeroelasticity and Structural Dynamics, Stockholm, Sweden, June 2007.

[14] C. Mettot, D. Sipp, and H. Bézard, "Quasi-laminar stability and sensitivity analyses for turbulent flows: Prediction of low-frequency unsteadiness and passive control," Journal of Physics of Fluids, vol. 26, no. 045112, April 2014.

[15] A. Lepage, Y. Amosse, D. Le Bihan, C. Poussot-Vassal, V. Brion, and E. Rantet, "A complete experimental investigation of gust load: from generation to active control," in Proceedings of the International Forum on Aeroelasticity and Structural Dynamics, Saint Petersbourg, Russia, June 2015.

[16] L. Burlion, C. Poussot-Vassal, P. Vuillemin, M. Leitner, and T. Kier, "Longitudinal Manoeuvre Load Control of a Flexible Large-Scale Aircraft," in Proceedings of the IFAC World Congress, Cap Town, South Africa, August 2014, pp. 3413-3418.

[17] A. J. Mayo and A. C. Antoulas, "A framework for the solution of the generalized realization problem," Linear Algebra and its Applications, vol. 425 , no. 2, pp. 634-662, 2007.

[18] C. Ionita, "Lagrange rational interpolation and its applications to approximation of large-scale dynamical systems," Ph.D. dissertation, University of Houston, Texas, USA, 2013

[19] A. Antoulas, S. Lefteriu, and A. Ionita, Model reduction and approximation theory and algorithms, ser. SIAM, Philadelphia. P. Benner, A. Cohen, M. Ohlberger and K. Willcox Eds, 2016, ch. A tutorial introduction to the Loewner framework for model reduction.

[20] P. Apkarian and D. Noll, "Nonsmooth $\mathcal{H}_{\infty}$ Synthesis," IEEE Transaction on Automatic Control, vol. 51, no. 1, pp. 71-86, January 2006.

[21] F. Demourant and C. Poussot-Vassal, "A new frequency-domain subspace algorithm with restricted poles location through LMI regions and its application to a wind tunnel test," International Journal of Control, to appear 2016.

[22] S. Gugercin, A. C. Antoulas, and C. A. Beattie, " $\mathcal{H}_{2}$ Model Reduction for Large Scale Linear Dynamical Systems," SIAM Journal on Matrix Analysis and Applications, vol. 30, no. 2, pp. 609-638, June 2008

[23] P. Vuillemin, C. Poussot-Vassal, and D. Alazard, "Poles Residues Descent Algorithm for Optimal Frequency-Limited $\mathcal{H}_{2}$ Model Approximation," in Proceedings of the 13th European Control Conference, Strasbourg, France, June 2014, pp. 1080-1085.

[24] C. Poussot-Vassal and P. Vuillemin, "Introduction to MORE: a MOdel REduction Toolbox," in Proceedings of the IEEE Multi-conference on Systems and Control, Dubrovnik, Croatia, October 2012, pp. 776-781.

[25] Z. Drmac, S. Gugercin, and C. Beattie, "Vector fitting for matrix-valued rational approximation," arXiv:1503.00411, 2015.

[26] — , "Quadrature-Based Vector Fitting For Discretized $\mathcal{H}_{2}$ Approximation," SIAM Journal on Scientific Computing, vol. 37, no. 2, pp. 625-652, 2015.

[27] T. McKelvey, H. Akcay, and L. Ljung, "Subspace-based multivariable system identification from frequency response data," IEEE transactions on Automatic Control, vol. 41, no. 7, pp. 960-979, Jul 1996.

[28] J. Maciejowski, "Guaranteed stability with subspace methods," Systems \& Control Letters, vol. 26, no. 2, pp. 153 - 156, 1995.

[29] N. Chui and J. Maciejowski, "Realization of stable models with subspace methods," Automatica, vol. 32, no. 11, pp. 1587 - 1595, 1996.

[30] T. V. Gestel, J. A. K. Suykens, P. V. Dooren, and B. D. Moor, "Identification of stable models in subspace identification by using regularization," IEEE transactions on Automatic Control, vol. 46, no. 9, pp. 1416-1420, Sep 2001.

[31] S. L. Lacy and D. S. Bernstein, "Subspace identification with guaranteed stability using constrained optimization," IEEE transactions on Automatic Control, vol. 48, no. 7, pp. 1259-1263, July 2003.

[32] K. Liu, R. Jacques, and D. Miller, "Frequency domain structural system identification by observability range space extraction," Journal of Dynamic Systems, Measurement, and Control, vol. 118, no. 2, pp. 211-220, Jun 1996.

[33] M. Chilali and P. Gahinet, " $\mathcal{H}_{\infty}$ design with pole placement constraints: an LMI approach," IEEE transactions on Automatic Control, vol. 41, no. 3, pp. 358-367, Mar 1996.
[34] M. Chilali, P. Gahinet, and P. Apkarian, "Robust pole placement in LMI regions," IEEE transactions on Automatic Control, vol. 44, no. 12, pp. 2257-2270, Dec 1999. 